\title{
Money non-neutrality in a Rational Belief Equilibrium with financial assets. ${ }^{\star}$
}

\author{
Maurizio Motolese \\ Istituto di Politica Economica, Università Cattolica di Milano, via Necchi 5, 20123 \\ Milano, Italy (e-mail: maurizio.motolese@mi.unicatt.it)
}

\begin{abstract}
In Rational Beliefs Equilibria money is generically non-neutral. Given the expectational perspective proposed by the Theory of Rational Belief Equilibrium, we show that one of the most important factors in the emergence of money non-neutrality is played by Endogenous Uncertainty. This, in contrast to the Rational Expectations results of money neutrality and policy ineffectiveness, leads to a scenario in which monetary policy has an impact on the real economy and price volatility. The heterogeneity of beliefs together with the distribution and intensity of agents' states of optimism/pessimism can amplify the real effect of monetary policy and/or generate endogenous fluctuations in the economy which are not explained by any exogenous shock. We claim that money non-neutrality is mostly an expectations driven phenomenon. Indeed, additional assumptions of asymmetry of information and/or unanticipated monetary policy are not needed to explain the real effect of monetary policy as it is customary in the New Classical Theory.
\end{abstract}

Key words Money non-neutrality - Monetary Policy - Rational Expectations - Rational Beliefs - Rational Belief Equilibrium - Endogenous Uncertainty - States of Belief

JEL Classification Numbers C68, D5, D84, E52

\footnotetext{
* I would like to thank Mordecai Kurz for his constant help and support. I have also greatly benefited from helpful discussions with Stanley Black, Kenneth L. Judd, Carsten Nielsen, Karl Schmedders and Ho-Mou Wu and from very useful comments of an anonymous referee. All errors are of course my own. Financial support from Fondazione ENI Enrico Mattei, Milano, Italy, and Fondazione Cassa di Risparmio di Piacenza e Vigevano, Piacenza, Italy, is gratefully acknowledged.
} 


\section{Introduction.}

One of the most recurrent themes in monetary theory is that of Money Neutrality. For several hundred years (dating back to David Hume [12] in the $17^{\text {th }}$ century) economists have argued whether changes in money supply have any effect on real variables like production, consumption, employment or real wages. A huge amount of empirical and theoretical research has been produced and yet the issue is still debated.

The recent view regarding the neutrality of money is from the New Classical Macroeconomics School. The neutrality theory, developed by Lucas [25], Sargent and Wallace [31] and often referred to as LSW proposition, states that any anticipated monetary shock would have no effect on real economic variables neither in the short run nor in the long run. Based on their theory of Rational Expectations, New Classical economists believe in the idea of policy irrelevance. In other words, since individuals hold Rational Expectations, they conclude that fully anticipated monetary policies are ineffective in the short run, as well as in the long run; only unanticipated policy shocks can influence real variables in the short run while in the long run the classical neutrality proposition holds. In a model in which the stock of money is exogenous, under the LSW proposition, the growth rate of money, assumed exogenous as well and fully perceived by agents, is the sole determinant of the rate of inflation: money is said to be dynamically neutral.

In Rational Beliefs Equilibria (RBE) money is generically non-neutral. Given the expectational perspective proposed by the Theory of RBE, we show that one of the most important factors in the emergence of money non-neutrality is played by Endogenous Uncertainty ${ }^{1}$. This, in contrast to the Rational Expectations results of money neutrality and policy ineffectiveness, leads to a scenario in which monetary policy has an impact on the real economy and price volatility. Under Rational Beliefs agents have different beliefs and different predictions about the effect of any particular monetary policy. Such heterogeneity of beliefs together with the distribution and intensity of agents' states of optimism/pessimism can amplify the real effect of monetary policy and/or generate endogenous fluctuations in the economy which are not explained by any exogenous shock. In the model under study in this paper the exogenous monetary growth rate is not the sole determinant of the inflation rate. States of belief amplify or decrease the effect of monetary policy on price level fluctuations leading to a more volatile pattern of inflation rates than under the Macro Rational Expectations hypothesis and thus making money dynamically non-neutral. We claim that money non-neutrality is mostly an expectations driven phenomenon. Indeed, additional assumptions of asymmetry of information and/or unanticipated

1 "Endogenous Uncertainty is that component of price volatility which is caused by the distribution of beliefs" (Kurz and Motolese [23], p. 13). For an exhaustive definition of Endogenous Uncertainty and a discussion of its emergence and role in equilibrium see also Kurz [17], [20] and [21]. 
monetary policy are not needed to explain the real effect of monetary policy as it is customary in the New Classical Theory.

The Rational Expectations approach dominated almost all economic thought in the 1970's and early 1980's. However, a growing stream of research in the 1980's has been devoted to the issue of policy effectiveness and many examples have been constructed to demonstrate the theoretical possibility of non-neutrality of money even when agents anticipate changes in money supply (see Fisher [7],[8] and Phelps and Taylor [30]). Chiappori and Guesnerie [5] proves the existence of a new class of non-linear solutions to the Lucas' [25] model which exhibit the non-neutrality property. Concern about the validity of the LSW proposition has also been raised by the theoretical sunspots literature. Azariadis [1], Azariadis and Guesnerie [2], Cass and Shell [4] and others show that money can be non-neutral in a wide class of models in which equilibrium depends on the realization of such random variables as sunspots which have no inherent relevance to the fundamentals of the economy.

The main bulk of the New Classical Macroeconomics rests on the assumption that all agents hold Rational Expectations. In order to explain the empirical evidence of money non-neutrality the New Classical theorists introduce asymmetric information which becomes the driving force of the theory: agents are assumed to be unable to observe information which is public in other parts of the economy. In this paper we show that money nonneutrality can arise endogenously and one way to do this is to allow agents to hold heterogeneous beliefs which are rationally formed in the sense of the Theory of Rational Beliefs developed by Kurz [18], [19] ${ }^{2}$.

This paper also addresses the issue of finding numerical solution to a Rational Belief infinite horizon monetary economy. The exogenous stochastic processes of money growth and dividends along with the endogenous state of belief of agents completely determine the transition probability of the economy from date $t$ to date $t+1$. The stochastic structure of the economy is Markov and we are interested in the numerical solutions of such an economy. In computing equilibria we follow closely the work of Judd, Kubler and Schmedders [15] who developed a computational procedure based on the B-spline collocation methods of approximating the equilibrium price and portfolio policy functions. In order to study the dynamic behavior of the economy, time series are generated via MonteCarlo simulations.

The rest of the paper is organized as follows. Section 2 presents the model of a standard infinite horizon pure monetary exchange economy and defines both the Rational Expectations Equilibrium (REE) and the RBE. Section 3 describes the computational procedure used to numerically solve for equilibria of the model in section 2. Section 4 reports and discusses the simulated results of money neutrality/non-neutrality and the effects of monetary policy both in REE and in RBE. Section 5 concludes.

\footnotetext{
${ }^{2}$ The effect of heterogeneous beliefs on equilibrium variables has also been studied by Harrison and Kreps [10], Varian [33] and Harris and Raviv [9].
} 


\section{The model.}

We consider a standard infinite horizon pure monetary exchange economy with a single, homogeneous, consumption good and two infinitely lived agents. Each one of them receives at the beginning of each period an endowment (reflecting labor output) of the commodity. Additional output, as in Lucas [26] is exogenously introduced in the economy by a production firm which is owned by the agents. The profit of the firm is produced according to the stationary Markov process $\left\{D_{t}, t=1,2, \ldots\right\}$, that will be specified below. The ownership of the shares, which we call stock, is a long-lived asset in aggregate unit net supply. In the financial structure of the economy, beside the stock market, a market for fiat money exists. Fiat money is issued by a government and is transferred to the agents at the beginning of each period. The transfer is assumed to be proportional to the pre-transfer holdings of the agents. Let $M_{t-1}$ denote the aggregate pre-transfer money holdings, that is the aggregate money purchased by the agents at $t-1$ and carried over next period at date $t$. Then the aggregate post-transfer money holdings at date $t$ are given by the equation

$$
M_{t}=M_{t-1} x_{t}
$$

where $\left\{x_{t}, t=1,2, \ldots\right\}$ is a stationary Markov process that will be specified below. Each agent's initial endowment of the two assets is denoted by $\pi_{0}^{k}=$ $\left(\theta_{0}^{k}, M_{0}^{k}\right), k=1,2 .{ }^{3}$ Note that our modeling of money follows the "Chicago" tradition in economic literature of using helicopter money as opposed to the most realistic open market operations money. The notation which we employ is as follows: for $k=1,2$

$$
\begin{array}{ll}
C_{t}^{k} & \text { - consumption of agent } k \text { at } t \\
\theta_{t}^{k} & \text { - stock holdings of agent } k \text { at } t \\
M_{t}^{k} & \text { - money holdings of agent } k \text { at } t \\
P_{t} & \text { - price of consumption good at date } t \\
\tilde{q}_{t} & \text { - price of common stock at } t \\
\Omega_{t}^{k} & \text { - endowment of agent } k \text { at } t \\
D_{t} & \text { - total amount of dividends produced exogenously at } t \\
M_{t} & \text { - money supply at date } t \\
H_{t} & \text { - history of all observable up to } t \\
u^{k}(\cdot, \cdot) & \text { - the utility function of agent } k .
\end{array}
$$

Each agent $k$ has von-Neumann-Morgensten preferences which are defined by a strictly monotone $C^{2}$ concave utility function $u^{k}: \mathbb{R}_{++} \times \mathbb{R}_{++} \rightarrow$ $\mathbb{R}$ and a discount factor $\beta_{k} \in(0,1)$. For any consumption sequence $C^{k}=$

\footnotetext{
${ }^{3}$ It would be straightforward to extend the theoretical study of this model to the case of several infinitely lived assets and several agents. However, since we are interested in the numerical solutions of such model, we are constrained by computational feasibility and we chose not to introduce further assets or agents. In fact, this would increase the number of state-variables and cause various technical problems for our computations.
} 
$\left\{C_{t}^{k}\right\}_{t=0}^{\infty}$ and real money balances sequence $M^{k}=\left\{M_{t}^{k} / P_{t}\right\}_{t=0}^{\infty}$ the associated utility for agent $k$ is therefore:

$$
U^{k}\left(C^{k}, M^{k}\right)=E\left\{\sum_{t=0}^{\infty} \beta_{k}^{t} u^{k}\left(C_{t}^{k}, \frac{M_{t}^{k}}{P_{t}}\right)\right\} .
$$

\subsection{The Equilibrium Concept.}

The optimization problem of agent $k$ for $k=1,2$ has the following common structure at all $t=1,2, \ldots$ :

$$
\max _{\left(C^{k}, \theta^{k}, M^{k}\right)} E_{Q_{t}^{k}}\left\{\sum_{\tau=t}^{\infty} \beta_{k}^{\tau-1} u^{k}\left(C_{\tau}^{k}, \frac{M_{\tau}^{k}}{P_{\tau}}\right) \mid H_{t}\right\} .
$$

subject to

$$
\begin{aligned}
& P_{t} C_{t}^{k}+\tilde{q}_{t} \theta_{t}^{k}+M_{t}^{k}=P_{t} \Omega_{t}^{k}+\theta_{t-1}^{k}\left(\tilde{q}_{t}+P_{t} D_{t}\right)+M_{t-1}^{k} x_{t} \\
& C_{t}^{k}, M_{t}^{k} \geq 0 \\
& \pi_{0}^{k}=\left(\theta_{0}^{k}, M_{0}^{k}\right) \text { given. }
\end{aligned}
$$

To enable us to compute the various equilibria in this chapter we assume that the common utility function of the two agents has the following functional form

$$
\frac{1}{1-\gamma_{k}}\left(C_{t}^{k}\right)^{1-\gamma_{k}}+\frac{1}{1-\nu_{k}}\left(\frac{M_{t}^{k}}{P_{t}}\right)^{1-\nu_{k}} \quad \gamma_{k}, \nu_{k}>0 .
$$

With this specification the Euler equations of agent $k$ for $k=1,2$ are

$$
\begin{gathered}
-\frac{\tilde{q}_{t}}{P_{t}}\left(C_{t}^{k}\right)^{-\gamma_{k}}+\beta_{k} E_{Q_{t}^{k}}\left(\left(C_{t+1}^{k}\right)^{-\gamma_{k}}\left(\frac{\tilde{q}_{t+1}}{P_{t+1}}+D_{t+1}\right) \mid H_{t}\right)=0 \\
-\frac{1}{P_{t}}\left(C_{t}^{k}\right)^{-\gamma_{k}}+\frac{1}{P_{t}}\left(\frac{M_{t}^{k}}{P_{t}}\right)^{-\nu_{k}}+\beta_{k} E_{Q_{t}^{k}}\left(\left(C_{t+1}^{k}\right)^{-\gamma_{k}}\left(\frac{x_{t+1}}{P_{t+1}}\right) \mid H_{t}\right)=0
\end{gathered}
$$

and the market clearing conditions are

$$
\begin{gathered}
\theta_{t}^{1}+\theta_{t}^{2}=1 \\
M_{t}^{1}+M_{t}^{2}=M_{t} .
\end{gathered}
$$

We assume that the economy is Markovian where the exogenous process of dividends $\left\{D_{t}, t=1,2, \ldots\right\}$ is a stationary and ergodic Markov process. The state space of the process is $\Delta=\left\{D^{H}, D^{L}\right\}$ with transition matrix

$$
\left[\begin{array}{cc}
\vartheta & 1-\vartheta \\
1-\vartheta & \vartheta
\end{array}\right]
$$


We refer to $D^{H}\left(D^{L}\right)$ as the high (low) dividend states.

The exogenous growth process of money $\left\{x_{t}, t=1,2, \ldots\right\}$ is also a stationary and ergodic Markov process. The state space of the process is $X=\left\{x^{H}, x^{L}\right\}$ with transition matrix

$$
\left[\begin{array}{cc}
\chi & 1-\chi \\
1-\chi & \chi
\end{array}\right]
$$

We refer to $x^{H}\left(x^{L}\right)$ as the loose (tight) monetary policy states. Throughout the paper we assume that $x_{t}$ are observable at date $t$. This assumption needs to be evaluated in relation to the assumption made by Lucas [25] and we comment on this later.

To ensure stability in the sense of Kurz [18] let us define $p_{t}=P_{t} / M_{t}^{4}$, $q_{t}=\tilde{q}_{t} / M_{t}$ and let $z_{t}^{k}=M_{t}^{k} / M_{t}$ be the proportion of the total money supply held by agent $k$ at $t$.

In equilibrium the inflation rates for all transitions from $t$ to $t+1$ are defined by

$$
i_{t+1}=\frac{P_{t+1}-P_{t}}{P_{t}} \quad t=1,2, \ldots
$$

and using the definition, given above, of $p_{t}$ and equation (1) we redefine them by

$$
i_{t+1}=\frac{x_{t+1} p_{t+1}-p_{t}}{p_{t}} \quad t=1,2, \ldots
$$

Given the definitions above we then rewrite the maximization problem of agent $k$ for $k=1,2$ at all $t$ as follows:

$$
\max _{\left(C^{k}, \theta^{k}, z^{k}\right)} E_{Q_{t}^{k}}\left\{\sum_{\tau=t}^{\infty} \beta_{k}^{\tau-1}\left(\frac{1}{1-\gamma_{k}}\left(C_{\tau}^{k}\right)^{1-\gamma_{k}}+\frac{1}{1-\nu_{k}}\left(\frac{z_{\tau}^{k}}{p_{\tau}}\right)^{1-\nu_{k}}\right) \mid H_{t}\right\} .
$$

subject to

$$
\begin{aligned}
& p_{t} C_{t}^{k}+q_{t} \theta_{t}^{k}+z_{t}^{k}=p_{t} \Omega_{t}^{k}+\theta_{t-1}^{k}\left(q_{t}+p_{t} D_{t}\right)+z_{t-1}^{k} \\
& C_{t}^{k}, z_{t}^{k} \geq 0 \\
& \pi_{0}^{k}=\left(\theta_{0}^{k}, z_{0}^{k}\right) \text { given. }
\end{aligned}
$$

The Euler equations of agent $k$ for $k=1,2$ are

$$
-\frac{q_{t}}{p_{t}}\left(C_{t}^{k}\right)^{-\gamma_{k}}+\beta_{k} E_{Q_{t}^{k}}\left(\left(C_{t+1}^{k}\right)^{-\gamma_{k}}\left(\frac{q_{t+1}}{p_{t+1}}+D_{t+1}\right) \mid H_{t}\right)=0
$$

\footnotetext{
4 To simplify notation in the rest of the paper we call the ratios $p_{t}=P_{t} / M_{t}$, "prices".
} 


$$
-\frac{1}{p_{t}}\left(C_{t}^{k}\right)^{-\gamma_{k}}+\frac{1}{p_{t}}\left(\frac{z_{t}^{k}}{p_{t}}\right)^{-\nu_{k}}+\beta_{k} E_{Q_{t}^{k}}\left(\left(C_{t+1}^{k}\right)^{-\gamma_{k}}\left(\frac{1}{p_{t+1}}\right) \mid H_{t}\right)=0
$$

and the market clearing conditions are

$$
\begin{aligned}
& \theta_{t}^{1}+\theta_{t}^{2}=1 \\
& z_{t}^{1}+z_{t}^{2}=1 .
\end{aligned}
$$

Under our assumption on agents' preferences the Euler equations of both agents together with the market clearing conditions fully characterize a competitive equilibrium.

Note that $x_{t}$ disappeared from the budget constraint of the agent. This is a direct result of the assumption that $x_{t}$ is observed and hence given $M_{t-1}$ the knowledge of $M_{t}$ and $x_{t}$ are equivalent. We find it easier to write the system in terms of $M_{t}$ rather than $x_{t}$. However, expectations of $M_{t+1}$ must be based on the conditional expectations of $x_{t+1}$ since $M_{t+1}=M_{t} x_{t+1}$. But then the information about $x_{t}$ is indeed part of the system (15)-(18) since $x_{t}$ is part of the conditioning $H_{t}$ in equation (13).

We shall continue to assume that changes in the money supply are uniform across agents.

Definition 1 A Markov Competitive Equilibrium with initial portfolio holdings $\left(\pi_{0}^{1}, \pi_{0}^{2}\right)$ is a stochastic process of prices, portfolios and exogenous shocks $\left\{\left(p_{t}, q_{t}, \pi_{t}^{1}, \pi_{t}^{2}, D_{t}, x_{t}\right), t=1,2, \ldots\right\}$ with the specified initial portfolio at $t=0$ such that

(i) these random variables satisfy, at all dates $t$, equations (14),(15)-(16) and the market clearing conditions (17)-(18);

(ii) there exist two sequences of functions $\left(\xi_{t}^{1}, \xi_{t}^{2}\right)$ such that the optimal portfolios satisfy the Markovian condition $\pi_{t}^{k}=\xi_{t}^{k}\left(D_{t}, x_{t}, \pi_{t-1}^{k}, p_{t}, q_{t}\right) \quad k=$ 1,2 ;

(iii) the price process $\left\{\left(p_{t}, q_{t}\right), t=1,2, \ldots\right\}$ is defined by a sequence of functions $\left[\begin{array}{c}p_{t} \\ q_{t}\end{array}\right]=\Phi_{t}\left(D_{t}, x_{t}, \pi_{t-1}^{1}, \pi_{t-1}^{2}\right)$.

The time dependence of the functions in definition 1 represents the potential time dependence of the beliefs of the agents. In the case of REE there are no states of beliefs which affect prices and all functions are not time dependent. More specifically, given the price map in (iii) we compute recursive equilibria in our infinite horizon economy assuming that the individuals' portfolios holdings $\left(\pi_{t}^{1}, \pi_{t}^{2}\right)$ are functions of $\left(\pi_{t-1}^{1}, \pi_{t-1}^{2}\right)$, the exogenous shocks $D_{t}$ and $x_{t}$, and of the endogenous state of beliefs. We follow the usual assumption in the applied literature (see for example Telmer [32], Heaton and Lucas [11] or Judd, Kubler and Schmedders [14],[15]) of asserting that the exogenous state and the agents' portfolio holdings constitute a sufficient statistic for the evolution of the infinite horizon economy. 
In any RBE such minimal state space is enlarged to include the endogenous state of beliefs. We also postulate the existence of continuous policy functions $f$ and price functions $g$ which map last period's portfolio holdings, the current endogenous state of beliefs and the exogenous shocks into the current portfolio holdings and asset prices, respectively. No conditions on the fundamentals of the economy are known to ensure existence of a recursive equilibrium (see Judd, Kubler and Schmedders [14],[15] for a detailed discussion) and, as it standard in the applied literature, we assume the existence of such an equilibrium. We further comment on this set of assumptions later.

We now review the REE case before turning to the more general examination of RBE.

2.1.1 Rational Expectations Equilibria. In any REE $Q^{1}=Q^{2}$ and there is no state of beliefs to effect prices. Since we seek a Markov equilibrium, the conditional probabilities of $\left(p_{t+1}, q_{t+1}, D_{t+1}, x_{t+1}\right)$ in (15)-(16) are conditioned only on the realized value of $D_{t}$ and $x_{t}$, and on the portfolios at date $t$, namely $\left(\pi_{t}^{1}, \pi_{t}^{2}\right)$. It then follows that the demand functions must take the form

$$
\begin{aligned}
& z_{t}^{k}=z^{k}\left(D_{t}, x_{t}, \pi_{t-1}^{k}, p_{t}, q_{t}\right) \\
& \theta_{t}^{k}=\theta^{k}\left(D_{t}, x_{t}, \pi_{t-1}^{k}, p_{t}, q_{t}\right) .
\end{aligned}
$$

(19)-(20) and the market clearing conditions (17)-(18) imply the equilibrium map

$$
\left[\begin{array}{c}
p_{t} \\
q_{t}
\end{array}\right]=\widehat{\Phi}\left(D_{t}, x_{t}, \pi_{t-1}^{1}, \pi_{t-1}^{2}\right) .
$$

Equation (21) shows that the transition function of the equilibrium Markov process can be described in the following way. Given the date $t-1$ portfolios $\left(\pi_{t-1}^{1}, \pi_{t-1}^{2}\right)$, there are only four vectors of prices $\left(p_{t}, q_{t}\right)$ that may occur at date $t$ :

$$
\begin{aligned}
& \left(p_{t}^{H H}, q_{t}^{H H}\right) \text { associated with }\left(D_{t}^{H}, x_{t}^{H}\right) \\
& \left(p_{t}^{H L}, q_{t}^{H L}\right) \text { associated with }\left(D_{t}^{H}, x_{t}^{L}\right) \\
& \left(p_{t}^{L H}, q_{t}^{L H}\right) \text { associated with }\left(D_{t}^{L}, x_{t}^{H}\right) \\
& \left(p_{t}^{L L}, q_{t}^{L L}\right) \text { associated with }\left(D_{t}^{L}, x_{t}^{L}\right) \text {. }
\end{aligned}
$$

Hence, there are only four distinct prices which may conditionally occur at any date and the transition probabilities from prices at $t$ to prices at $t+1$ are exactly equal to the joint transitions from $\left(D_{t}, x_{t}\right)$ to $\left(D_{t+1}, x_{t+1}\right)$. That is, given any portfolio $\left(\pi_{t}^{1}, \pi_{t}^{2}\right)$ at date $t$, the time invariant transition matrix of prices is

\begin{tabular}{c|c|c|c|c|} 
& $\left(p_{t+1}^{H H}, q_{t+1}^{H H}\right)$ & $\left(p_{t+1}^{H L}, q_{t+1}^{H L}\right)$ & $\left(p_{t+1}^{L H}, q_{t+1}^{L H}\right)$ & $\left(p_{t+1}^{L L}, q_{t+1}^{L L}\right)$ \\
\hline$\left(p_{t}^{H H}, q_{t}^{H H}\right)$ & $\vartheta \chi$ & $\vartheta(1-\chi)$ & $(1-\vartheta) \chi$ & $(1-\vartheta)(1-\chi)$ \\
\hline$\left(p_{t}^{H L}, q_{t}^{H L}\right)$ & $\vartheta(1-\chi)$ & $\vartheta \chi$ & $(1-\vartheta)(1-\chi)$ & $(1-\vartheta) \chi$ \\
\hline$\left(p_{t}^{L H}, q_{t}^{L H}\right)$ & $(1-\vartheta) \chi$ & $(1-\vartheta)(1-\chi)$ & $\vartheta \chi$ & $\vartheta(1-\chi)$ \\
\hline$\left(p_{t}^{L L}, q_{t}^{L L}\right)$ & $(1-\vartheta)(1-\chi)$ & $(1-\vartheta) \chi$ & $\vartheta(1-\chi)$ & $\vartheta \chi$ \\
\hline
\end{tabular}


Indeed, as we show later, given that $p_{t}=P_{t} / M_{t}, x_{t}$ are observed and money is neutral under Rational Expectations it follows that at each date $t$, $\left(p_{t}^{H H}, q_{t}^{H H}\right)=\left(p_{t}^{H L}, q_{t}^{H L}\right)$ and $\left(p_{t}^{L H}, q_{t}^{L H}\right)=\left(p_{t}^{L L}, q_{t}^{L L}\right)$. Only two prices are realized at any date $t$, the monetary shock has no impact and the transitions from prices at $t$ to prices at $t+1$ are entirely governed by the real shock process in the economy. The transition probabilities are then exactly equal to the joint transitions from $D_{t}$ to $D_{t+1}$. That is, given any portfolio $\left(\pi_{t}^{1}, \pi_{t}^{2}\right)$ at date $t$, the time invariant transition matrix of prices is

\begin{tabular}{c|c|c|} 
& $\left(p_{t+1}^{H}, q_{t+1}^{H}\right)$ & $\left(p_{t+1}^{L}, q_{t+1}^{L}\right)$ \\
\hline$\left(p_{t}^{H}, q_{t}^{H}\right)$ & $\vartheta$ & $1-\vartheta$ \\
\hline$\left(p_{t}^{L}, q_{t}^{L}\right)$ & $1-\vartheta$ & $\vartheta$ \\
\hline
\end{tabular}

In comparison with Lucas' [25] finite horizon model, if $x_{t}$ are observed, full neutrality is attained under Rational Expectations. Thus, our methodology is to assume that $x_{t}$ are observed and confirm that full neutrality is realized even under infinite horizon. However, under the same assumption we shall show later that neutrality is not preserved under Rational Beliefs even when $x_{t}$ are observed. One of the reasons for the non-neutrality result in RBE's, as we shall see in details later, is that the endogenous unobserved states of belief are part of the true state space of the economy which causes the market structure to be dynamically incomplete. In the symmetric REE under study, in which agents are identical, the absence of asymmetric information and the money neutrality result cause the market structure to be dynamically complete.

We now turn to the examination of the structure of beliefs.

2.1.2 The structure of beliefs. The development here uses concepts from the Theory of Rational Beliefs for which a clear explanation can be found in Kurz [20],[18], and [19].

We use the tools of assessment variables to construct Markov RBE as developed in Kurz and Schneider [24]. Assessment variables are parameters indicating how the agent perceives the state of the economy and are thus tools for the description of stable and non-stationary processes. From a purely formal point of view, the assessment variables of the two agents are simply two infinite sequences of random variables which we denote by $\left\{y_{t}^{k}, t=1,2, \ldots\right\}$ for $k=1,2$. The variables may be correlated. In our application, $y_{t}^{k} \in Y=\{1,0\}$. Assessment variables attain their significance when we formulate the way agents' beliefs vary in response to different realization of these variables. In general one may think of the belief of agent $k$ as a probability on the joint process $\left\{\left(p_{t}, q_{t}, D_{t}, x_{t}, y_{t}^{k}\right), t=1,2, \ldots\right\}$. However, all dynamic decisions of agent $k$ are made with the conditional probability of $\left\{\left(p_{t}, q_{t}, D_{t}, x_{t}\right), t=1,2, \ldots\right\}$ given the value taken by $y_{t}^{k}$. In the equilibria formulated below $\left\{\left(p_{t}, q_{t}, D_{t}, x_{t}, y_{t}^{k}\right), t=1,2, \ldots\right\}$ is a Markov process and 
hence $y_{t}^{k}$ are allowed to be interdependent with other variables. The conditional probabilities of $\left(p_{t+1}, q_{t+1}, D_{t+1}, x_{t+1}\right)$ given $\left(p_{t}, q_{t}, D_{t}, x_{t}\right)$ will also be a Markov process but it will be non-stationary; it will utilize different matrices depending upon $y_{t}^{k}$.

Since $y_{t}^{k}$ exhausts all the non-stationarity perceived by the agents, we consider policy functions of the time invariant form

$$
\begin{aligned}
& \theta_{t}^{k}=\theta^{k}\left(D_{t}, x_{t}, y_{t}^{k}, \pi_{t-1}^{k}, p_{t}, q_{t}\right) \\
& z_{t}^{k}=z^{k}\left(D_{t}, x_{t}, y_{t}^{k}, \pi_{t-1}^{k}, p_{t}, q_{t}\right) .
\end{aligned}
$$

Note that in equations (15)-(16) agent $k$ specifies the probability of $\left(p_{t+1}, q_{t+1}, D_{t+1}, x_{t+1}, y_{t+1}^{k}\right)$ conditional on $\left(p_{t}, q_{t}, D_{t}, x_{t}, y_{t}^{k}, \pi_{t-1}^{k}\right)$ - the value taken by his assessment variable jointly with the observed data. Given that $\theta_{t+1}^{k}$ and $z_{t+1}^{k}$ are as in (22)-(23) it follows from our Markov assumptions that the demands of agent $k$ for stocks and money are functions of exactly the form specified in (22)-(23), which we then take to be the optimal policies. Consequently we can write the market clearing conditions as

$$
\begin{aligned}
& \theta^{1}\left(D_{t}, x_{t}, y_{t}^{1}, \pi_{t-1}^{1}, p_{t}, q_{t}\right)+\theta^{2}\left(D_{t}, x_{t}, y_{t}^{2}, \pi_{t-1}^{2}, p_{t}, q_{t}\right)=1 \\
& z^{1}\left(D_{t}, x_{t}, y_{t}^{1}, \pi_{t-1}^{1}, p_{t}, q_{t}\right)+z^{2}\left(D_{t}, x_{t}, y_{t}^{2}, \pi_{t-1}^{2}, p_{t}, q_{t}\right)=1 .
\end{aligned}
$$

Agent $k$ 's demand functions for stocks and money (22)-(23) do not depend on the other agent's assessment variable. Assessment variables are privately perceived parameters indicating how an agent interprets current information. These variables have purely subjective meaning and do not represent objective and transferable "information".

The system (24)-(25) implies that the equilibrium map of this economy takes the form

$$
\left[\begin{array}{c}
p_{t} \\
q_{t}
\end{array}\right]=\Phi^{*}\left(D_{t}, x_{t}, y_{t}^{1}, y_{t}^{2}, \pi_{t-1}^{1}, \pi_{t-1}^{2}\right)
$$

The equilibrium map (26) reveals that conditioning on the portfolios at $t-1$, equilibrium prices are determined by the exogenous shocks $D_{t}$ and $x_{t}$, and by the state of belief represented by the vector of assessment variables $\left(y_{t}^{1}, y_{t}^{2}\right)$. We assumed that $y_{t}^{k} \in Y=\{1,0\}$, and will later define the beliefs so that "1" will be a state of optimism with respect to high dividend states at date $t+1$ while " 0 " will be a state of pessimism. In the way we specify the assessment variables, they completely specify the conditional probability beliefs of the agents at any date.

The equilibrium map (26) implies that given each pair of portfolios $\left(\pi_{t-1}^{1}, \pi_{t-1}^{2}\right)$, there are at most 16 distinct price vectors $\left(p_{t}, q_{t}\right)$ that may occur at date $t$ and these correspond to the 16 combinations of $\left(D_{t}, x_{t}, y_{t}^{1}, y_{t}^{2}\right)$. Moreover, due to our Markov assumption and given any portfolios $\left(\pi_{t}^{1}, \pi_{t}^{2}\right)$, the true, equilibrium, transition probability from the 16 prices $\left(p_{t}, q_{t}\right)$ to 
the 16 prices $\left(p_{t+1}, q_{t+1}\right)$ (which are likely to be different from prices at $t$ ) is determined entirely by the transition probability from $\left(D_{t}, x_{t}, y_{t}^{1}, y_{t}^{2}\right)$ to $\left(D_{t+1}, x_{t+1}, y_{t+1}^{1}, y_{t+1}^{2}\right)$.

We select the stochastic joint process $\left\{\left(D_{t}, x_{t}, y_{t}^{1}, y_{t}^{2}\right), t=1,2, \ldots\right\}$ to be a stationary Markov process with a transition matrix $\Gamma$ which is independent of $\left(\pi_{t}^{1}, \pi_{t}^{2}\right)$. It follows from the equilibrium map (26) that, although the values of prices at date $t+1$ depend upon the portfolios $\left(\pi_{t}^{1}, \pi_{t}^{2}\right)$, the true probability of transition from $\left(p_{t}, q_{t}\right)$ to $\left(p_{t+1}, q_{t+1}\right)$ is $\Gamma$ at all $t$, independent of $\left(\pi_{t}^{1}, \pi_{t}^{2}\right)$. This choice ${ }^{5}$ implies that the true equilibrium process of prices $\left\{\left(p_{t}, q_{t}\right), t=1,2, \ldots\right\}$, is a stationary process with a fixed transition probability from $\left(p_{t}, q_{t}\right)$ to $\left(p_{t+1}, q_{t+1}\right)$ defined by $\Gamma$. Since this is a stationary transition it follows from the ergodic theorem that the agents, who compute the empirical distribution of the equilibrium process, will discover $\Gamma$ and this matrix will be used to construct the stationary measure. We then say that the matrix $\Gamma$ characterizes the stationary measure of the equilibrium dynamics.

The optimum conditions (15)-(16) require each agent to forecast prices $\left(p_{t+1}, q_{t+1}\right)$ and his own portfolio next period. Since the forecasting of his own portfolio is done conditional upon market prices at $t$, this task is within the range of behavior that we can expect a rational agent to perform. A more complex issue is the determination, at time $t$, of the set of 16 prices $\left\{\left(p_{t+1}, q_{t+1}\right)\right\}$, that can be realized at date $t+1$, given the date $t$ portfolios $\left(\pi_{t}^{1}, \pi_{t}^{2}\right)$ and prices $\left(p_{t}, q_{t}\right)$. We assume that all agents know this set of prices based on the past history of the equilibrium process. ${ }^{6}$

We can now clarify the role of assessment variables. In (22)-(23) we specified that the demand functions are not time dependent and hence the

\footnotetext{
5 The equilibrium dynamics are generated by a fixed and stationary matrix. This choice has been a matter of convenience and simplicity. The process $\left\{\left(D_{t}, x_{t}, y_{t}^{1}, y_{t}^{2}\right), t=1,2, \ldots\right\}$ could have been selected to be any stable process with Markov stationary measure induced by the empirical distribution. In such a case the fixed transition matrix $\Gamma$ would characterize only the stationary measure of the equilibrium dynamics rather than be the matrix of the true probability of the equilibrium dynamics of prices.

${ }^{6}$ The theory of rational beliefs is based on the premise of abundant set of past data to reveal anything which can be statistically revealed. The problem here is that the model has infinite number of prices and the relative frequency of any particular price is zero for most prices. It is then true that the agents could look at all past dates at which the given configuration of portfolios $\left(\theta_{t}^{1}, z_{t}^{1}, \theta_{t}^{2}, z_{t}^{2}\right)$ and prices $\left(p_{t}, q_{t}\right)$ occurred in the past to determine the set of 16 prices that will occur at $t+1$. The problem is that the frequency at which any particular configuration of prices and portfolio occurs is zero. Agents could then disagree about the set of prices that may occur at $t+1$. The assumption that the set of possible prices is known to the agents is important for the simplification of the computations in the following section. We may also note that even when agents know the set of possible prices, they may still disagree upon the probabilities at which these prices will occur and that depends upon the value which their assessment variable takes at date $t$.
} 
assessment variables completely determine the conditional probabilities of the agents. But from the assumption of a Markov equilibrium it must be that an assessment variable $y_{t}^{k}$ must determine completely the transition matrix from $\left(p_{t}, q_{t}\right)$ to $\left(p_{t+1}, q_{t+1}\right)$ which is perceived by agent $k$ at date $t$. Moreover, $y_{t}^{k} \in Y=\{1,0\}$ implies that the agent has at most two $16 \times 16$ Markov matrices and at each date the value taken by his assessment variable determines which of these two the agent uses.

The equilibrium map (26) implies that $\left(\mathbb{R}^{2} \times \mathbb{R}^{2} \times \Delta \times X \times Y \times Y\right)^{\infty}$ is the state space for prices. However, one can also think of the state space as $\left(\mathbb{R}^{2} \times \mathbb{R}^{2} \times S\right)^{\infty}$ where $S$ is simply the price index space $S=\{1,2, \ldots, 16\}$. We then define a new equilibrium map $\Phi$ between the indexes of prices and the states of dividends, monetary policy and assessment variables by

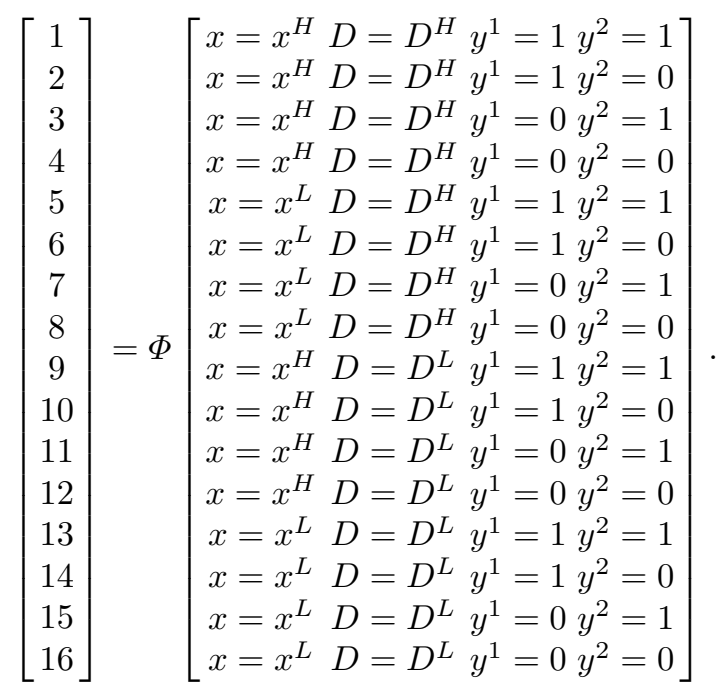

The maps (26) and (27) highlight the definition of Endogenous Uncertainty which identifies the variability of prices at a given state of the exogenous variables.

We finally turn to a technical issue that needs clarification. The reader may have noted earlier that we defined agent $k$ 's belief $Q^{k}$ as a probability on the space of infinite sequences $\left\{\left(D_{t}, x_{t}, y_{t}^{k}, \pi_{t}^{1}, \pi_{t}^{2}, p_{t}, q_{t}\right), t=1,2, \ldots\right\}$. However, we have shown that conditioning on the given portfolios $\left(\pi_{t}^{1}, \pi_{t}^{2}\right)$ at $t$, the belief of an agent was reduced to selecting a transition matrix from $\left(p_{t}, q_{t}\right)$ to $\left(p_{t+1}, q_{t+1}\right)$. Thus, we assumed that agents know the partition of the price state space $\left(\mathbb{R}^{2} \times \mathbb{R}^{2} \times \Delta \times X \times Y \times Y\right)^{\infty}$ induced by the distribution of the exogenous variables. Note that this assumption implies that the earlier specified states of optimism (pessimism) with respect to high(low) dividend states at date $t+1$ represent also states of optimism (pessimism) about the occurrence of the associated stock prices. Since the high(low) dividend states are those of the higher(lower) stock prices agents are thus optimistic(pessimistic) with respect to high(low) stock prices at date $t+1$. 
The beliefs $Q^{1}$ and $Q^{2}$ of the two agents, given portfolios $\left(\pi_{t}^{1}, \pi_{t}^{2}\right)$ at date $t$, are represented by Markov transition matrices from $\left(p_{t}, q_{t}, y_{t}^{k}\right)$ to $\left(p_{t+1}, q_{t+1}, y_{t+1}^{k}\right)$. The probability used in (15)-(16) is $Q^{k}\left((\cdot) \mid y^{k}\right)$, the probability which is conditional on the assessment variable. The rationality of belief conditions requires that

(i) $Q^{k}\left((\cdot) \mid y^{k}\right)$ is a stable measure and the dynamics of the economy under it has an empirical distribution with probability 1 ;

(ii) the stationary measure of $Q^{k}\left((\cdot) \mid y^{k}\right)$ equals the probability on infinite sequences induced by the true transition matrix $\Gamma$.

Since $Q^{k}\left((\cdot) \mid y^{k}\right)$ is represented by the two Markov matrices used by the agent at different times, we need to specify the joint distribution of $\left(p_{t}, q_{t}, y_{t}^{k}\right)$ and the implied rationality conditions which are consistent with these Markov matrices. To do that we use the Conditional Stability Theorem (see Kurz and Schneider [24] p. 494). It says that if the probability $Q^{k}$ of the joint process $\left\{\left(p_{t}, q_{t}, y_{t}^{k}\right), t=1,2, \ldots\right\}$ is stable, then $Q^{k}\left((\cdot) \mid y^{k}\right)$ is a stable probability on $\left\{\left(p_{t}, q_{t}\right), t=1,2, \ldots\right\}$ and the stationary measure of $Q^{k}\left((\cdot) \mid y^{k}\right)$ is the marginal of $Q^{k}$ on $\left(p_{t}, q_{t}\right)$ obtained by integrating on $y^{k}$.

For simplicity in analysis and computations, we assume the marginal distribution of $Q^{k}$ on $y_{t}^{k}$ to be i.i.d. and denote this unconditional probability by $Q^{k}\left(\left\{y_{t}^{k}=1\right\}\right)=\alpha_{k}$ for $k=1,2$. The Conditional Stability Theorem then implies that the beliefs of the two agents are described by two pairs of matrices, $\left(F^{1}, F^{2}\right)$ for agent 1 and $\left(G^{1}, G^{2}\right)$ for agent 2 , such that $Q^{1}$ and $Q^{2}$ are characterized by the following conditions:

$$
\begin{array}{ll}
Q^{1} \text { for agent 1: } & \text { adopt } F^{1} \text { if } y_{t}^{1}=1 \\
& \text { adopt } F^{2} \text { if } y_{t}^{1}=0 \\
& \alpha_{1} F^{1}+\left(1-\alpha_{1}\right) F^{2}=\Gamma \\
& \\
Q^{2} \text { for agent 1: } \quad & \text { adopt } G^{1} \text { if } y_{t}^{2}=1 \\
& \text { adopt } G^{2} \text { if } y_{t}^{2}=0 \\
& \alpha_{2} G^{1}+\left(1-\alpha_{2}\right) G^{2}=\Gamma
\end{array}
$$

where $\alpha_{1}$ is the frequency at which agent 1 uses matrix $F^{1}$ and $\alpha_{2}$ is the frequency at which agent 2 uses matrix $G^{1}$.

We proceed to construct the RBE by assembling the conditions which the Markov transition matrix $\Gamma$ has been assumed to satisfy. The matrix $\Gamma$ must satisfy the following

the marginal on $y_{t}^{k}$ is i.i.d. with $P\left\{y_{t}^{1}=1\right\}=\alpha_{k}$;

the marginal on $D_{t}$ is Markov as specified by (9)

the marginal on $x_{t}$ is Markov as specified by (10)

the joint distribution of $\left(y_{t}^{1}, y_{t}^{2}\right)$ may depend upon $D_{t}$ and $x_{t}$. 
The following matrix satisfies all the conditions specified in (30)-(33):

$$
\Gamma=\left[\begin{array}{cccc}
\vartheta \chi A & \vartheta(1-\chi) A & (1-\vartheta) \chi A & (1-\vartheta)(1-\chi) A \\
\vartheta(1-\chi) B & \vartheta \chi B & (1-\vartheta)(1-\chi) B & (1-\vartheta) \chi B \\
(1-\vartheta) \chi C & (1-\vartheta)(1-\chi) C & \vartheta \chi C & \vartheta(1-\chi) C \\
(1-\vartheta)(1-\chi) D & (1-\vartheta) \chi D & \vartheta(1-\chi) D & \vartheta \chi D
\end{array}\right]
$$

where $(A, B, C, D)$ are all $4 \times 4$ matrices characterized by the 18 parameters $\left(\alpha_{1}, \alpha_{2}, a, b, c, d\right), a=\left(a_{1}, a_{2}, a_{3}, a_{4}\right), b=\left(b_{1}, b_{2}, b_{3}, b_{4}\right), c=\left(c_{1}, c_{2}, c_{3}, c_{4}\right)$, $d=\left(d_{1}, d_{2}, d_{3}, d_{4}\right)$ and of the following type:

$$
A=\left[\begin{array}{cccc}
a_{1} & \alpha_{1}-a_{1} & \alpha_{2}-a_{1} & 1+a_{1}-\alpha_{1}-\alpha_{2} \\
a_{2} & \alpha_{1}-a_{2} & \alpha_{2}-a_{2} & 1+a_{2}-\alpha_{1}-\alpha_{2} \\
a_{3} & \alpha_{1}-a_{3} & \alpha_{2}-a_{3} & 1+a_{3}-\alpha_{1}-\alpha_{2} \\
a_{4} & \alpha_{1}-a_{4} & \alpha_{2}-a_{4} & 1+a_{4}-\alpha_{1}-\alpha_{2}
\end{array}\right]
$$

(35) implies that $P\left\{y_{t}^{1}=1\right\}=\alpha_{k}$ for $k=1,2$ as required by (30). Note, however, that although each process $\left\{y_{t}^{k}, t=1,2, \ldots\right\}$ for $k=1,2$ is very simple, the joint process $\left\{\left(D_{t}, x_{t}, y_{t}^{1}, y_{t}^{2}\right), t=1,2, \ldots\right\}$ may be complex: it allows correlation among the four variables and these effects could be important. This joint effect of the assessment variables, as distinct from the individually perceived effect, is part of $\Gamma$ which describes the externalities of beliefs in the market performance. These externalities are reproduced in the equilibrium process. They are aimed to specify those interactions among agents which reflect the structure of communications in society. To complete the definition of $Q^{1}$ and $Q^{2}$ in (28-29) it is left to specify matrices $\left(F^{1}, F^{2}, G^{1}, G^{2}\right)$. Select 32 parameters $\lambda=\left(\lambda_{1}, \lambda_{2}, \ldots, \lambda_{16}\right)$ and $\mu=\left(\mu_{1}, \mu_{2}, \ldots, \mu_{16}\right)$ which will be interpreted later, and define the row vectors of $A$ with the notation

$$
A^{j}=\left(a_{j}, \alpha_{1}-a_{j}, \alpha_{2}-a_{j}, 1+a_{j}-\left(\alpha_{1}+\alpha_{2}\right)\right) j=1,2,3,4 .
$$

Similar notation is used for $B, C$ and $D$. We then construct the family of rational beliefs which we use in the simulations by defining eight matrix functions of $z=\left(z_{1}, z_{2}, \ldots, z_{16}\right)$ :

$$
A_{1}(z)=\left[\begin{array}{c}
z_{1} A^{1} \\
z_{2} A^{2} \\
z_{3} A^{3} \\
z_{4} A^{4}
\end{array}\right], \quad A_{2}^{\vartheta}(z)=\left[\begin{array}{l}
\frac{1-\vartheta z_{1}}{1-\vartheta} A^{1} \\
\frac{1-\vartheta z_{2}}{1-\vartheta} A^{2} \\
\frac{1-\vartheta z_{3}}{1-\vartheta} A^{3} \\
\frac{1-\vartheta z_{4}}{1-\vartheta} A^{4}
\end{array}\right]
$$




$$
\begin{gathered}
B_{1}(z)=\left[\begin{array}{l}
z_{5} B^{1} \\
z_{6} B^{2} \\
z_{7} B^{3} \\
z_{8} B^{4}
\end{array}\right], B_{2}^{\vartheta}(z)=\left[\begin{array}{l}
\frac{1-\vartheta z_{5}}{1-\vartheta} B^{1} \\
\frac{1-\vartheta z_{6}}{1-\vartheta} B^{2} \\
\frac{1-\vartheta z_{7}}{1-\vartheta} B^{3} \\
\frac{1-\vartheta z_{8}}{1-\vartheta} B^{4}
\end{array}\right] \\
C_{1}(z)=\left[\begin{array}{l}
z_{9} C^{1} \\
z_{10} C^{2} \\
z_{11} C^{3} \\
z_{12} C^{4}
\end{array}\right], C_{2}^{\vartheta}(z)=\left[\begin{array}{l}
\frac{1-(1-\vartheta) z_{9}}{1-(1-\vartheta) z_{10}} C^{1} \\
\frac{1-(1-\vartheta) z_{11}}{\vartheta} C^{3} \\
\frac{1-(1-\vartheta) z_{12}}{\vartheta} C^{4}
\end{array}\right] \\
D_{1}(z)=\left[\begin{array}{l}
z_{13} D^{1} \\
z_{14} D^{2} \\
z_{15} D^{3} \\
z_{16} D^{4}
\end{array}\right], D_{2}^{\vartheta}(z)=\left[\begin{array}{l}
\frac{1-(1-\vartheta) z_{13}}{\vartheta} D^{1} \\
\frac{1-(1-\vartheta) z_{14}}{\vartheta} D^{2} \\
\frac{1-(1-\vartheta) z_{15}}{\vartheta} D^{3}
\end{array}\right] .
\end{gathered}
$$

Hence given the definitions (37)-(40) we define matrices $\left(F^{1}(\lambda), F^{2}(\lambda)\right)$ for $\lambda=\left(\lambda_{1}, \lambda_{2}, \ldots, \lambda_{16}\right)$ by

$$
F^{1}(\lambda)=\left[\begin{array}{cccc}
\vartheta \chi A_{1}(\lambda) & \vartheta(1-\chi) A_{1}(\lambda) & (1-\vartheta) \chi A_{2}^{\vartheta}(\lambda) & (1-\vartheta)(1-\chi) A_{2}^{\vartheta}(\lambda) \\
\vartheta(1-\chi) B_{1}(\lambda) & \vartheta \chi B_{1}(\lambda) & (1-\vartheta)(1-\chi) B_{2}^{\vartheta}(\lambda) & (1-\vartheta) \chi B_{2}^{\vartheta}(\lambda) \\
(1-\vartheta) \chi C_{1}(\lambda) & (1-\vartheta)(1-\chi) C_{1}(\lambda) & \vartheta \chi C_{2}^{\vartheta}(\lambda) & \vartheta(1-\chi) C_{2}^{\vartheta}(\lambda) \\
(1-\vartheta)(1-\chi) D_{1}(\lambda) & (1-\vartheta) \chi D_{1}(\lambda) & \vartheta(1-\chi) D_{2}^{\vartheta}(\lambda) & \vartheta \chi D_{2}^{\vartheta}(\lambda)
\end{array}\right]
$$

and $F^{2}(\lambda)$ is then defined by the rationality condition of beliefs

$$
F^{2}(\lambda)=\frac{1}{1-\alpha_{1}}\left(\Gamma-\alpha_{1} F^{1}(\lambda)\right) .
$$

Finally given the definitions (37)-(40) we define matrices $\left(G^{1}(\mu), G^{2}(\mu)\right)$ for $\mu=\left(\mu_{1}, \mu_{2}, \ldots, \mu_{16}\right)$ by

$$
G^{1}(\mu)=\left[\begin{array}{cccc}
\vartheta \chi A_{1}(\mu) & \vartheta(1-\chi) A_{1}(\mu) & (1-\vartheta) \chi A_{2}^{\vartheta}(\mu) & (1-\vartheta)(1-\chi) A_{2}^{\vartheta}(\mu) \\
\vartheta(1-\chi) B_{1}(\mu) & \vartheta \chi B_{1}(\mu) & (1-\vartheta)(1-\chi) B_{2}^{\vartheta}(\mu) & (1-\vartheta) \chi B_{2}^{\vartheta}(\mu) \\
(1-\vartheta) \chi C_{1}(\mu) & (1-\vartheta)(1-\chi) C_{1}(\mu) & \vartheta \chi C_{2}^{\vartheta}(\mu) & \vartheta(1-\chi) C_{2}^{\vartheta}(\mu) \\
(1-\vartheta)(1-\chi) D_{1}(\mu) & (1-\vartheta) \chi D_{1}(\mu) & \vartheta(1-\chi) D_{2}^{\vartheta}(\mu) & \vartheta \chi D_{2}^{\vartheta}(\mu)
\end{array}\right]
$$


and $G^{2}(\mu)$ is then defined by the rationality condition of beliefs

$$
G^{2}(\mu)=\frac{1}{1-\alpha_{2}}\left(\Gamma-\alpha_{2} G^{1}(\mu)\right) .
$$

In order to ensure non-negative entries in the matrices $A, B, C$ and $D$ in (35), the selection of the vectors $(\lambda, \mu)$ is restricted by 96 inequality constraints which define the feasible region. These constraints are as follows:

$$
\begin{aligned}
& \lambda_{s} \leq \frac{1}{\vartheta} \quad \mu_{s} \leq \frac{1}{\vartheta} \quad \text { for } s=1,2, \ldots, 8 \\
& \lambda_{s} \leq \frac{1}{1-\vartheta} \quad \mu_{s} \leq \frac{1}{1-\vartheta} \quad \text { for } s=9,10, \ldots, 16 \\
& \lambda_{s} \leq \frac{1}{\alpha_{1}} \quad \mu_{s} \leq \frac{1}{\alpha_{2}} \quad \text { for } s=1,2, \ldots, 16 \\
& \lambda_{s} \geq \frac{\alpha_{1}+\vartheta-1}{\vartheta \alpha_{1}} \mu_{s} \geq \frac{\alpha_{2}+\vartheta-1}{\vartheta \alpha_{2}} \text { for } s=1,2, \ldots, 8 \\
& \lambda_{s} \geq \frac{\alpha_{1}-\vartheta}{(1-\vartheta) \alpha_{1}} \mu_{s} \geq \frac{\alpha_{2}-\vartheta}{(1-\vartheta) \alpha_{2}} \text { for } s=9,10, \ldots, 16 \text {. }
\end{aligned}
$$

To motivate the construction of the family of rational beliefs above note that the parameters $\lambda_{s}$ and $\mu_{s}$ are multiplied by the rows of $A, B, C$ and $D$. They proportionally change the conditional probabilities of the four sets of four states $(1,2,3,4),(5,6,7,8),(9,10,11,12)$ and $(13,14,15,16)$ relative to the stationary measure represented by the matrix $\Gamma$ in (34). Since $\lambda_{s}$ and $\mu_{s}$ are the factors of proportionality by which agents' conditional probability beliefs deviate from the stationary probabilities in $\Gamma$, we refer to them as intensity parameters. So far agents' assessment variables have been used to endogenously enlarge the price state space and no actual economic meaning has been attached to them. They attain meaning only when the agents specify how they interpret these variables in generating their conditional probability beliefs. For example, $\lambda_{s}>1$ implies increased probabilities of states $(1,2,3,4)$ and $(5,6,7,8)$ in $F^{1}$ relative to $\Gamma$ given that agent 1 is in state $s$. This means that the assessment variables induce more optimism or pessimism about the occurrence of states at $t+1$ relative to $\Gamma$. Given the equilibrium map (27), the family of Markovian Rational Beliefs constructed above allows any revision of the conditional probabilities of states of high dividend exogenous shock $(1,2,3,4,5,6,7,8)$ relative to the stationary measure $\Gamma$, to be offset by an opposite direction revision of the conditional probabilities of states of low dividend exogenous shock $(9,10,11,12,13,14,15,16)$.

\subsubsection{Rational Belief Equilibria.}


Definition $2 A$ Markov RBE is a Markov Competitive Equilibrium such that:

(i) associated with each date $t$ optimal portfolio $\left\{\left(\pi_{t}^{1}, \pi_{t}^{2}\right), t=1,2, \ldots\right\}$ there exist feasible sets of date $t+1$ prices $J\left(\pi_{t}^{1}, \pi_{t}^{2}\right)$ which are known to the agents, each consisting of, at most, 16 prices;

(ii) each agent holds a Markov rational belief of the following form: at each date $t$ he uses one of two $16 \times 16$ price transition matrices $\left(F^{1}, F^{2}\right)$ for agent 1 and $\left(G^{1}, G^{2}\right)$ for agent 2 in accord with his assessment variable and the rationality conditions (28)-(29). All four matrices are Markov transition matrices from $J\left(\pi_{t-1}^{1}, \pi_{t-1}^{2}\right)$ to $J\left(\pi_{t}^{1}, \pi_{t}^{2}\right)$;

(iii) optimal portfolios take the form $\pi_{t}^{k}=\xi^{k}\left(D_{t}, x_{t}, y_{t}^{k}, \pi_{t-1}^{k}, p_{t}, q_{t}\right) \quad k=$ 1,2 and the equilibrium map has the form (26).

To clarify definition 2 we need to note what the agent is assumed to know. He clearly does not know the equilibrium map (26). Indeed, he does not observe the assessment variable of other agents and thus could not learn the equilibrium map. In the optimization (2)-(3) we assume that at date $t-1$ the portfolio $\left(\pi_{t-1}^{1}, \pi_{t-1}^{2}\right)$ is observed by all agents hence all know the set of possible prices $J\left(\pi_{t-1}^{1}, \pi_{t-1}^{2}\right)$ that may occur at date $t$. At date $t$ the agents use transition matrices from the 16 prices $\left(p_{t}, q_{t}\right)$ in $J\left(\pi_{t-1}^{1}, \pi_{t-1}^{2}\right)$ which are possible at $t$ to the 16 prices $\left(p_{t+1}, q_{t+1}\right)$ in $J\left(\pi_{t}^{1}, \pi_{t}^{2}\right)$ which are possible at $t+1$. As we just noted, they know $J\left(\pi_{t-1}^{1}, \pi_{t-1}^{2}\right)$ from date $t-1$ information and they know $J\left(\pi_{t}^{1}, \pi_{t}^{2}\right)$ from the fact that date $t$ market prices $\left(p_{t}, q_{t}\right)$ and portfolio $\left(\pi_{t}^{1}, \pi_{t}^{2}\right)$ are also observed at date $t$.

\section{Equilibrium price and portfolio policy functions.}

In computing equilibria of the infinite horizon monetary economy under study we follow closely the work of Judd, Kubler and Schmedders [14],[15] who developed a computational procedure, specified below, based on the B-spline collocation methods of approximating the equilibrium price and portfolio policy functions. Such a numerical algorithm allows agents to have a continuum of portfolios instead of allowing a discrete number of portfolios as it is in the approach used by Heaton and Lucas [11]. Heaton and Lucas [11] considered models with two agents and two securities and used a discretized version of the state space which tends to result in large approximation error. Indeed, they report error of up to 0.84 percent.

\subsection{The possible space and short sales cost function.}

As our model has two assets and two agents, the feasible space for the portfolio state variables is a subset of $\mathbb{R}^{4}$. However if in equilibrium the portfolio of agent 1 is $\pi_{t}^{1}=\left(\theta_{t}^{1}, z_{t}^{1}\right)$, the portfolio of agent 2 must be $\pi_{t}^{2}=\left(1-\theta_{t}^{1}, 1-z_{t}^{1}\right)$. Hence, focusing only on agent 1 we select a set 
$\Xi \subset \mathbb{R}^{2}$ to be the set of possible equilibrium portfolios $\pi_{t}^{1}=\left(\theta_{t}^{1}, z_{t}^{1}\right)$. To well approximate the equilibrium price and policy functions by splines with a finite number of nodes it is important to ensure that the endogenous set $\Xi$ is bounded. Agents are not allowed to take short positions in money holdings since, given the specifications of the model, it would result in allowing borrowing at zero cost (i.e. zero interest rate). On the other hand they can take short positions in stock holdings. Allowing agents to hold short positions causes some difficulties in the computational procedure when, out of equilibrium, in order to ensure positive consumption, agents may follow paths of unbounded sequences of short positions rendering the feasible set not compact. This motivated the use of holding cost of short positions. As for any unbounded sequence of short positions of agent 1 there is a correspondent unbounded sequence of long positions of agent 2 and vice versa, we also penalize any long position which exceeds the total supply of stock in the market. The feasible set is then bounded in both directions. The following cost function is then subtracted from the utility function of agent $k$ at date $t$ :

$$
\kappa\left(\theta_{t}^{k}\right)= \begin{cases}h / 4\left(\theta_{t}^{k}\right)^{4} & \text { if } \theta_{t}^{k}<0 \\ h / 4\left(\theta_{t}^{k}-1\right)^{4} & \text { if } \theta_{t}^{k}>1 \\ 0 & \text { otherwise }\end{cases}
$$

where $h \in \mathbb{R}_{+}$. With the set $\Xi$ being compact, the parameter $h$ may be selected so that the quartic holding cost function $\kappa\left(\theta_{t}^{k}\right)$ is very small inside the set but rises rapidly if an agent optimal choice is to move out of the set $\Xi$. Let $\kappa_{\theta}^{k}=\partial \kappa\left(\theta_{t}^{k}\right) / \partial \theta_{t}^{k}$. With this change in the utility function of agent $k$ we modify the Euler equations (15)-(16) to account for (46). Finally, we denote the set of possible equilibrium price vectors $\left(p_{t}, q_{t}\right)$ by the bounded set $\Upsilon$.

Recall that we denote by $\Delta \times X \times Y \times Y$ the state space of dividends, monetary shock and state of beliefs and by $S$ the set of indices of the 16 members of $\Delta \times X \times Y \times Y$. We have assumed that the current state of the dividends, the monetary shock and the assessment variables together with last period's portfolio provide sufficient information for an equilibrium. We have postulated the existence of the following continuous equilibrium functions

$$
\begin{gathered}
f^{k}=\left(f^{k \theta}, f^{k z}\right): S \times \Xi \Rightarrow \Xi \quad k=1,2 \\
g=(p, q): S \times \Xi \Rightarrow \Upsilon
\end{gathered}
$$

such that for all $s_{t} \in S$ and $\pi_{t-1}^{1} \in \Xi$, if the market clearing conditions are substituted into the Euler equations then these equations are satisfied with

$$
\pi_{t}^{k}=f^{k}\left(s_{t}, \pi_{t-1}^{k}, y_{t}^{k}\right)
$$


and

$$
\left(p_{t}, q_{t}\right)=g\left(s_{t}, \pi_{t-1}^{1}\right)
$$

The functions $f^{k}$ determine the optimal portfolio of agent $k$ given the prices at date $t$ (hence the state $s_{t}$ ), the assessment variable of agent $k$ at date $t$ and the portfolio at date $t-1$. The function $g$ determines the equilibrium prices at date $t$ given the state $s_{t}$ and the portfolio at date $t-1$.

We approximate the six functions $f$ and $g$ by two-dimensional tensor products of B-splines of order 4 (see Appendix A.2 for details) and in what follows we thus use $\hat{f}$ and $\hat{g}$ to denote such approximate policy functions.

\subsection{The new system of Euler Equations.}

Reconsider the budget equation (14), the Euler equations (15)-(16) and the functions (47)-(48). Given the price index state $s \in S=\{1,2, \ldots, 16\}$ and agent 1's beginning-of-period portfolio holdings $\pi_{-}^{1}=\left(\theta_{-}^{1}, z_{-}^{1}\right)$ we define agent 1's current period consumption by

$$
\begin{aligned}
C^{1}= & -\frac{\hat{g}^{q}\left(s, \pi_{-}^{1}\right)}{\hat{g}^{p}\left(s, \pi_{-}^{1}\right)} \hat{f}^{\theta}\left(s, \pi_{-}^{1}\right)-\frac{\hat{f}^{z}\left(s, \pi_{-}^{1}\right)}{\hat{g}^{p}\left(s, \pi_{-}^{1}\right)} \\
& +\Omega^{1}(s)+\theta_{-}^{1}\left(\frac{\hat{g}^{q}\left(s, \pi_{-}^{1}\right)}{\hat{g}^{p}\left(s, \pi_{-}^{1}\right)}+D(s)\right)+\frac{z_{-}^{1}}{\hat{g}^{p}\left(s, \pi_{-}^{1}\right)} .
\end{aligned}
$$

Let $\pi^{1}=\left(\hat{f}^{\theta}\left(s, \pi_{-}^{1}\right), \hat{f}^{z}\left(s, \pi_{-}^{1}\right)\right)$ denote agent 1's current portfolio and define his random next period consumption by

$$
\begin{aligned}
\widetilde{C}_{+}^{1}= & -\frac{\hat{g}^{q}\left(\tilde{s}, \pi^{1}\right)}{\hat{g}^{p}\left(\tilde{s}, \pi^{1}\right)} \hat{f}^{\theta}\left(\tilde{s}, \pi^{1}\right)-\frac{\hat{f}^{z}\left(\tilde{s}, \pi^{1}\right)}{\hat{g}^{p}\left(\tilde{s}, \pi^{1}\right)} \\
& +\Omega^{1}(\tilde{s})+\hat{f}^{\theta}\left(s, \pi_{-}^{1}\right)\left(\frac{\hat{g}^{q}\left(\tilde{s}, \pi^{1}\right)}{\hat{g}^{p}\left(\tilde{s}, \pi^{1}\right)}+D(\tilde{s})\right)+\frac{\hat{f}^{z}\left(s, \pi_{-}^{1}\right)}{\hat{g}^{p}\left(\tilde{s}, \pi^{1}\right)}
\end{aligned}
$$

Agent 2's current period consumption is defined by

$$
C^{2}=\Omega^{1}(s)+\Omega^{2}(s)+D(s)-C^{1}
$$

and his random next period consumption is defined by

$$
\widetilde{C}_{+}^{2}=\Omega^{1}(\tilde{s})+\Omega^{2}(\tilde{s})+D(\tilde{s})-\widetilde{C}_{+}^{1} .
$$


We thus obtain the following system of Euler equations for $k=1,2$

$$
\begin{aligned}
& \frac{\hat{g}^{q}\left(s, \pi_{-}^{1}\right)}{\hat{g}^{p}\left(s, \pi_{-}^{1}\right)}\left(C^{k}\right)^{-\gamma_{k}}+\kappa_{\theta}^{k} \\
= & +\beta_{k} \sum_{\tilde{s}=1}^{16}\left(\left(\widetilde{C}_{+}^{k}\right)^{-\gamma_{k}}\left(\frac{\hat{g}^{q}\left(\tilde{s}, \pi^{1}\right)}{\hat{g}^{p}\left(\tilde{s}, \pi^{1}\right)}+D(\tilde{s})\right)\right) Q^{k}\left(\tilde{s} \mid s, y^{k}\right) \\
& \frac{1}{\hat{g}^{p}\left(s, \pi_{-}^{1}\right)}\left(C^{k}\right)^{-\gamma_{k}}-\frac{1}{\hat{g}^{p}\left(s, \pi_{-}^{1}\right)}\left(\frac{\hat{f}^{k z}\left(s, \pi_{-}^{1}\right)}{\hat{g}^{p}\left(s, \pi_{-}^{1}\right)}\right)^{-\nu_{k}} \\
= & +\beta_{k} \sum_{\tilde{s}=1}^{16}\left(\left(\widetilde{C}_{+}^{k}\right)^{-\gamma_{k}}\left(\frac{1}{\hat{g}^{p}\left(\tilde{s}, \pi^{1}\right)}\right)\right) Q^{k}\left(\tilde{s} \mid s, y^{k}\right) .
\end{aligned}
$$

To compute the coefficients of the approximate policy functions $\hat{f}$ and $\hat{g}$ we select a grid $\mathcal{G}$ of mesh points $\pi_{-(i j)}^{1}=\left(\theta_{-(i)}^{1}, z_{-(j)}^{1}\right)_{i, j=1, \cdots, n}$. Now we take these points to be equal to $\pi_{-}^{1}$ and thus obtain 4 equations for each mesh point and each price index state $s \in S=\{1,2, \ldots, 16\}$. The resulting system is very large and has $n \times n \times 16 \times 4$ equations and unknowns $^{7}$. To solve the system we follow the Gauss-Jacobi approach used by Judd, Kubler and Schmedders [14],[15] in their spline collocation algorithm. We thus compute the approximate policy functions $\hat{f}$ and $\hat{g}$ through an iterative process starting with some initial guess $\hat{f}_{0}$ and $\hat{g}_{0}$. In each iteration $v=1,2, \cdots$, we then solve the system of Euler equations (53)-(54) ${ }^{8}$ at each mesh point $\pi_{-}^{1}=\left(\theta_{-}^{1}, z_{-}^{1}\right) \in \mathcal{G}$ and $s \in S$ by computing current portfolio decisions and asset prices given previous iteration functions $\hat{f}_{v-1}$ and $\hat{g}_{v-1}$ determining the policy process in the subsequent period. The coefficients characterizing the new set of functions $\hat{f}_{v}$ and $\hat{g}_{v}$ are finally obtained through interpolation. The procedure is discontinued when the maximal change in the value of the approximate policy functions, at all mesh points in successive iterations, is less then an error tolerance $\varepsilon$ : if $\max _{\pi \in \mathcal{G}, s \in S}\left\{\left|\hat{f}_{(v)}-\hat{f}_{(v-1)}\right|,\left|\hat{g}_{(v)}-\hat{g}_{(v-1)}\right|\right\}<\varepsilon$.

For a satisfactory approximation we use a collocation grid of size $15 \times 15$. And we set the error tolerance $\varepsilon=10^{-6}$ and require the error in the Euler equations to be less than $10^{-11}$ at each mesh point. However, in addition, our method examines the error at values of the state variables which are not used in the spline collocation procedure. For instance, we take a uniform sample of 400 points from the feasible set $\Xi$ and test the error of the Euler equations at those points. We then declare as solution any set of parameters for which the error of the Euler equation in the interior of $\Xi$ is less than $10^{-6}$. On points next to the boundary the consumption level of one of the

\footnotetext{
7 Note that the resulting system has the coefficients of the spline functions as unknowns.

8 The system of nonlinear equations has been solved by using a Newton based algorithm (see Appendix A.1 for details).
} 
agent $k, C^{k}$ and/or his held proportion of total money supply $z^{k}$ may be close to zero with very high marginal utility. Also, as we are close to the left/right part of the boundary (supposing stock holdings $\theta$ are on the $x$ axis), the holding cost increases. It is then clear that on this part of the boundary the computed errors in the Euler equations are not reliable. Judd, Kubler and Schmedders [14],[15] argue that on this part of the boundary we should not expect too high a precision. Moreover, given that in equilibrium the probability of the system reaching these areas is negligible, the larger errors in the Euler equations should not be a ground to reject the solution. We thus adopt the procedure of allowing a solution to have an error in the Euler equation of at most $10^{-4}$ on the boundaries.

\subsection{The parameterization.}

In all simulations we set the endowments to be $\Omega^{1}=\Omega^{2}=4$ all states $s$. To stress the role of endogenous uncertainty and state of beliefs we do not introduce additional heterogeneity among agents and therefore assume that $\beta_{1}=\beta_{2}=\beta, \gamma_{1}=\gamma_{2}=\gamma$ and $\nu_{1}=\nu_{2}=\nu$. Their values will be defined later case by case. We set the exogenous processes transition probabilities at $\vartheta=0.57$ and $\chi=0.4$. The exogenous dividend state space is set to be $\Delta=\left\{D^{H}, D^{L}\right\}=\{2.5,1.5\}$ and the money growth state space is set to be $X=\left\{x^{H}, x^{L}\right\}=\{1.02,0.98\}$.

REE are identified by the selection of $a_{s}=b_{s}=c_{s}=d_{s}=0.25$ for $s=1,2,3,4$. In the benchmark case of rational expectations the intensity parameters are required to be $\lambda_{s}=\mu_{s}=1$ for $s=1,2, \ldots, 16$.

In all RBE we set $\alpha_{1}=\alpha_{2}=0.57$ as in Kurz [22]. We set the parameters $a_{s}=b_{s}=c_{s}=d_{s}=0.15$ for $s=1,2,3,4$. The intensity parameters are set to be $\lambda_{s}=\mu_{s}=1.75$ for $s=1,2, \ldots, 16$.

Note that the above choice of parameter values does not aim to calibrate the model to simulate the behavior of any given country economy. We want only to show how money non-neutrality arises in a RBE. Our simulated economy represents an exercise in this sense. The belief intensity parameters $\lambda$ and $\mu$ have been chosen to be constant across states. This enable us to impose the condition of "anonymity" as defined in Kurz [22]. The endowment values have been set at a level which ensure strictly positive initial wealth to the agents for all points in the grid $\mathcal{G}$. This has also produced substantial savings in computational efforts and thus making convergence of our computations much smoother.

\section{Simulation results: on the non-neutrality of money.}

Our approach here is based on MonteCarlo simulations of the model under study. A simulation starts either from a specific portfolio or from a grid 
of portfolios and then iterated forward by selecting numbers from a random number generator (see Appendix A.3 for details) for the sequence of realizations of $\left\{\left(D_{t}, x_{t}, y_{t}^{1}, y_{t}^{2}\right), t=1,2, \ldots\right\}$.

The results reported below are focused on studying the characteristics of the joint effects of monetary policy and beliefs on inflation, real consumption, portfolio holdings and ergodic behavior of the economy.

We first note that, because the economy at study is ergodic (as we show below), the long term average inflation rate relative to the growth rate of money supply in the economy, both under REE and RBE is equal to zero.

Note also that at each date $t$ the economy is jointly affected by the exogenous monetary shocks $x_{t} \in X=\left\{x^{H}, x^{L}\right\}$, the endogenous states of beliefs $\left(y_{t}^{1}, y_{t}^{2}\right) \in Y \times Y=\{(1,1),(1,0),(0,1),(0,0)\}$, the real exogenous dividend shocks $D \in \Delta=\left\{D^{H}, D^{L}\right\}$ and agents' portfolio positions. It is clear that for any transition of the economy within each one of the real exogenous dividend regimes (i.e. when, due to the Markov assumption, $D_{t}=$ $D_{t+1}=D^{H}$ or $D_{t}=D_{t+1}=D^{L}$ ) prices and real variables are solely affected by the compound effect of exogenous monetary shocks, endogenous states of beliefs and agents' portfolio positions. On the other hand, for any transition of the economy between $D^{H}$ states and $D^{L}$ states, prices and real variables are also affected by the real exogenous dividend shocks. We want then to decompose the standard deviation of inflation rates and subtract from it the effect of the real exogenous dividend regimes. To do so we first compute the following two long term conditional inflation rates with fixed money supply (i.e. $x_{t}=1$ all $t$ ):

$$
\bar{i}_{H L}=E_{\Gamma}\left(i_{t+1} \mid D_{t+1}=D^{H}, D_{t}=D^{L}\right)
$$

and

$$
\bar{i}_{L H}=E_{\Gamma}\left(i_{t+1} \mid D_{t+1}=D^{L}, D_{t}=D^{H}\right) .
$$

Now let $\varpi_{t+1}^{H L}=1$ when $D_{t+1}=D^{H}, D_{t}=D^{L}$ and 0 otherwise, and let $\varpi_{t+1}^{L H}=1$ when $D_{t+1}=D^{L}, D_{t}=D^{H}$ and 0 otherwise. Hence that component of price level volatility, which is the solely joint effect of state of beliefs, monetary policy and agents' portfolio positions, will be defined by the standard deviation of the random variable

$$
\rho_{t+1}=i_{t+1}-\bar{i}_{H L} \varpi_{t+1}^{H L}-\bar{i}_{L H} \varpi_{t+1}^{L H} .
$$

Note that the policy functions $f$ and price functions $g$ are not independent of the agents' portfolio allocations. Under RBE the agents' endogenous state of beliefs strongly impacts the portfolio positions of the agents. Price and policy functions dependence on wealth distribution is a direct result of RBE. It is endogenously generated by the unobserved state of beliefs.

We simulate the economy under study and report in the tables below the standard deviation, min and max of inflation rates $\rho_{t}$ (specified in (55)); the conditional mean, standard deviation, min and max of agent 1's consumption level under the two exogenous dividend regimes $D_{t}=D^{H}$ and $D_{t}=D^{L}$. 
We now explore the characteristics of money neutrality and the effects of monetary policy in a symmetric REE ${ }^{9}$. In such an equilibrium agents are simply identical with $\beta=0.92, \gamma=3.25$ and $\nu=3.25$. Money neutrality is obtained and price and policy functions do not depend on wealth distribution as no trading takes place. Table 1 (column 1) reports the statistics for the REE. These results will provide a reference point for the study of the characteristics of money non-neutrality of RBE's. The results reported in Table 1 have been obtained by generating 225 simulations, each for 20,000 periods and recording the last 2,000 . We then compute the statistics on the obtained sample of 450,000 observations.

Table 1 Characteristics of money non-neutrality.

\begin{tabular}{llrr}
\hline & & REE & RBE \\
\hline std dev & $\rho_{t}$ & 0.020 & 0.026 \\
$\min$ & $\rho_{t}$ & -0.020 & -0.081 \\
$\max$ & $\rho_{t}$ & 0.020 & 0.076 \\
std dev & $x_{t}$ & 0.020 & 0.020 \\
avg & $C_{t}^{1} \mid D_{t}=D^{H}$ & 5.250 & 5.250 \\
std dev & $C_{t}^{1} \mid D_{t}=D^{H}$ & 0.000 & 0.723 \\
$\min$ & $C_{t}^{1} \mid D_{t}=D^{H}$ & 5.250 & 3.827 \\
$\max$ & $C_{t}^{1} \mid D_{t}=D^{H}$ & 5.250 & 6.665 \\
$\operatorname{avg}$ & $C_{t}^{1} \mid D_{t}=D^{L}$ & 4.750 & 4.750 \\
std dev & $C_{t}^{1} \mid D_{t}=D^{L}$ & 0.000 & 0.630 \\
$\min$ & $C_{t}^{1} \mid D_{t}=D^{L}$ & 4.750 & 3.497 \\
$\max$ & $C_{t}^{1} \mid D_{t}=D^{L}$ & 4.750 & 5.973 \\
\hline
\end{tabular}

Recall that we have assumed that the sequence $\left\{x_{t}, t=1,2, \ldots\right\}$ of money supply growth rates are observed. It is clear that in a REE money is dynamically neutral in two senses:

(i) any exogenous fluctuations in $x_{t}$ lead to exactly equal percentage changes in the price level;

(ii) all fluctuations in the price level induced by the observed fluctuations in $x_{t}$ have no real effects.

Given these observations we now proceed to explore the characteristics of money non-neutrality and the effects of monetary policy in asymmetric $\mathrm{RBE}^{10}$. In such equilibria agents are hence heterogeneous as they can hold different conditional probability beliefs at each date $t$.

\footnotetext{
${ }^{9}$ It would have been more efficient to solve for the REE using a Negishi approach. We instead used the B-spline collocation method because the REE policy function coefficients there obtained represented a good initial guess for computing RBE's.

10 In such equilibria agents have the same endowment and utility but hold different conditional probability beliefs in states of disagreement when $y_{t}^{1} \neq y_{t}^{2}$.
} 
In Table 1 (column 2) we report the statistical results for a RBE in which agents' utility function parameters $\beta, \gamma$ and $\nu$ are set at the same values specified for the REE in column 1.

In Figure 1 we report a sample of 100 observations of inflation rates $\rho_{t}$ (specified in (55)) and money growth rates $x_{t}$ out of a sample of 300,000 generated by the RBE of Table 1 (column 2) starting from the initial portfolio distribution $\pi_{0}^{1}=(0.5,0.5)$.

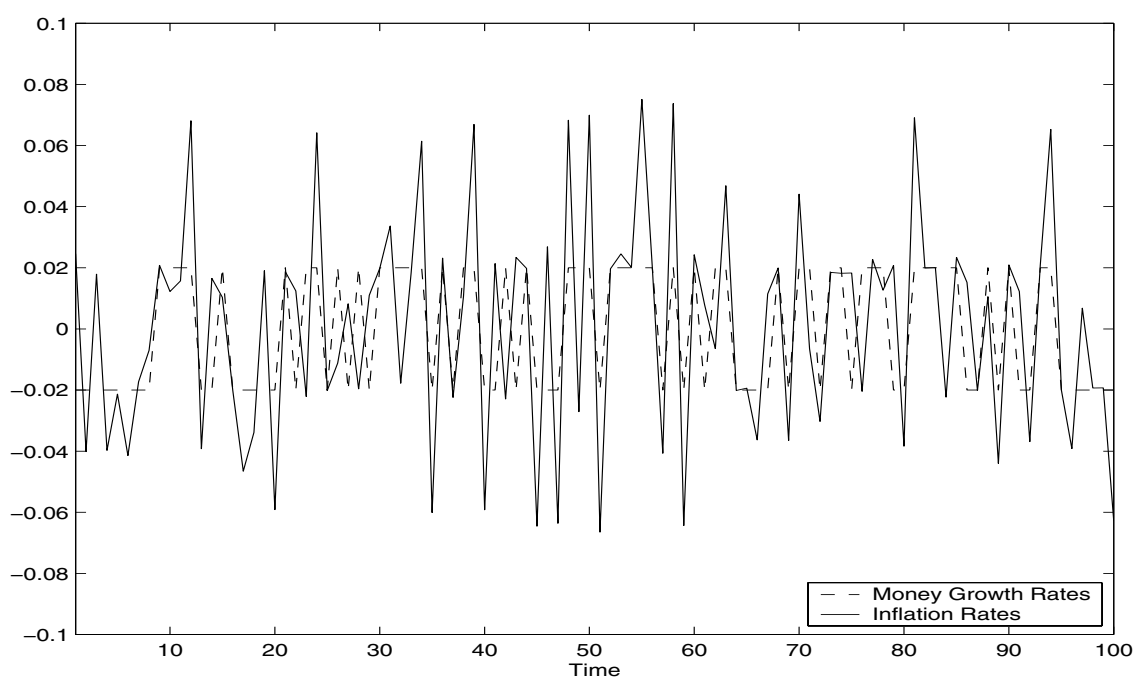

Fig. 1 Inflation rates and money growth rates in the $\operatorname{RBE} \beta=0.92, \gamma=3.25$ and $\nu=3.25$.

In Figure 2 we report a typical time series of agent 1's consumption level. We report 20,000 observations out of a sample of 300,000 generated by the RBE of Table 1 (column 2) starting from the initial portfolio distribution $\pi_{0}^{1}=(0.5,0.5)$.

We also report the RBE simulation results for $\gamma=\{1.5,3.25,5\}$ and $\nu=\{2.5,3.25,5\}$. Table 2 shows that the model exhibits a consistent and homogeneous impact of endogenous uncertainty on money non-neutrality for different values of agents' risk aversion parameters $\gamma$ and $\nu$. Similar results are also obtained varying agents' discount factor $\beta$ over the range $0.92-0.96$ and keeping $\gamma=3.25$ and $\nu=3.25$. We report them in Table 3 .

From Tables 1, 2 and 3 and Figures 1 and 2 one can see that, due to the presence of endogenous uncertainty, money is dynamically non-neutral under rational beliefs. Indeed, any exogenous fluctuations in the monetary growth rate $x_{t}$ do not necessarily lead to equal fluctuations in the inflation rate $\rho_{t}$. This can be checked from Figure 1 where the time series of inflation rates and the time series of monetary growth rates do not overlap. Furthermore, a higher price level volatility is observed. Such a higher volatility is 


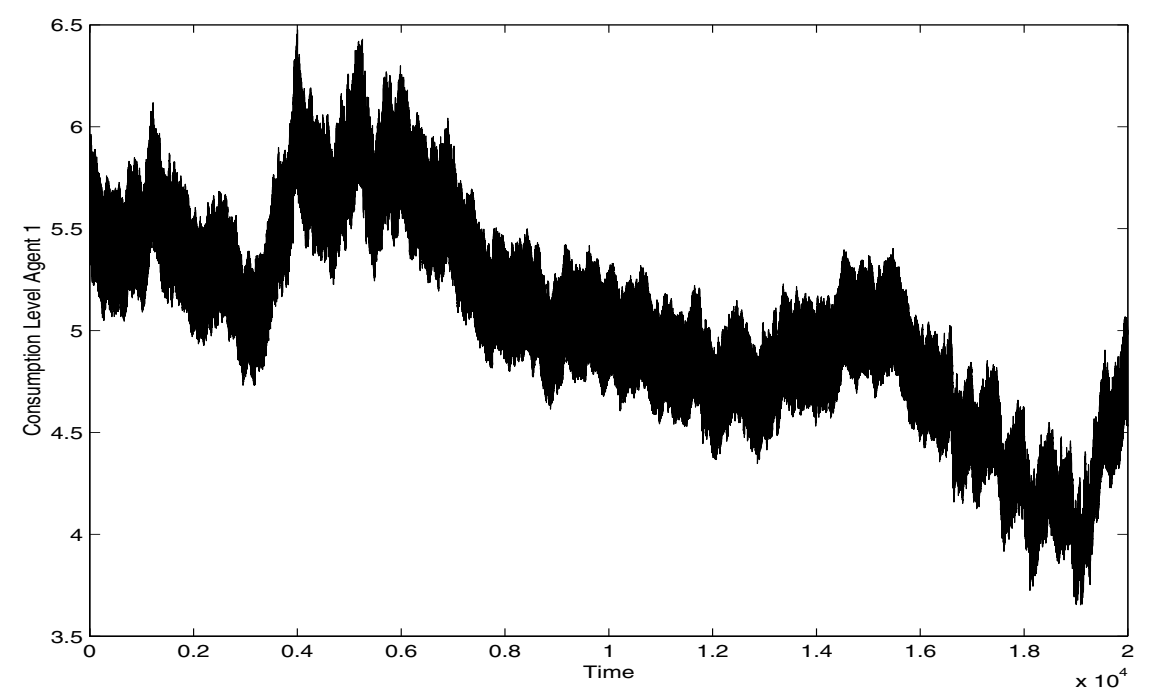

Fig. 2 Agent 1's consumption level in the $\operatorname{RBE} \beta=0.92, \gamma=3.25$ and $\nu=3.25$.

jointly induced by the growth rate of money and agents' conditional probability beliefs. It is also clear from Tables 1, 2 and 3 and Figure 2 that under Rational Beliefs monetary fluctuations have an impact on the real economy (for instance on agents' consumption allocation over time). While New Classical macroeconomists focus on ad hoc exogenous informational frictions to account for dynamic money non-neutrality we propose endogenous uncertainty, which is propagated within the economy by the beliefs and actions of agents, as the main driver of the real effects of monetary policy and money non-neutrality. The difference in conclusions results from the existence in our economy of unobserved states of belief which are part of the true state space and cause the market structure to be dynamically incomplete. Such component of uncertainty takes the form of additional prices induced by the states of belief and by their variability over time.

We now turn to explore the ergodic behavior of both the REE and RBE of Table 1. Note that the ergodic behaviour exhibited by the particular RBE of Table 1 is also a feature of all the RBE's of Tables 2 and 3. In Figure 3 we exhibit 450,000 REE portfolio allocations of agent 1 generated by an initial selection of 225 portfolios from a uniform grid $\mathcal{G}^{\prime} \subset \Xi$. For each initial point, we generated 2,000 iterations and recorded all of them.

Under REE the economy converges to a steady point characterized by a portfolio allocation $\pi^{1}=(0.5,0.5)$ and with consumption levels only depending on the exogenous dividend shocks. In fact, generating 1 million iterations for each one of the 225 initial points all the simulated portfolio holdings time series degenerate to the constant sequence $(0.5,0.5)$.

In Figure 4 we exhibit 450,000 portfolios of agent 1 in the asymmetric RBE of Table 1. The observations in Figure 4 were also generated by an 
Table 2 Characteristics of money non-neutrality.

\begin{tabular}{|c|c|c|c|c|c|c|}
\hline & & & & $\gamma=1.50$ & $\gamma=3.25$ & $\gamma=5.00$ \\
\hline \multirow{9}{*}{$\nu=2.50$} & std dev & $\rho_{t}$ & & 0.021 & 0.024 & 0.025 \\
\hline & $\min$ & $\rho_{t}$ & & -0.045 & -0.063 & -0.093 \\
\hline & $\max$ & $\rho_{t}$ & & 0.044 & 0.064 & 0.103 \\
\hline & std dev & $C_{t}^{1}$ & $D_{t}=D^{H}$ & 0.529 & 0.671 & 0.172 \\
\hline & $\min$ & $C_{t}^{1}$ & $D_{t}=D^{H}$ & 3.701 & 3.969 & 4.636 \\
\hline & $\max$ & $C_{t}^{1}$ & $D_{t}=D^{H}$ & 6.710 & 6.517 & 5.876 \\
\hline & std dev & $C_{t}^{1}$ & $D_{t}=D^{L}$ & 0.460 & 0.589 & 0.152 \\
\hline & $\min$ & $C_{t}^{1}$ & $D_{t}=D^{L}$ & 3.373 & 3.634 & 4.217 \\
\hline & $\max$ & $C_{t}^{1}$ & $D_{t}=D^{L}$ & 6.062 & 5.854 & 5.316 \\
\hline \multirow{9}{*}{$\nu=3.25$} & std dev & $\rho_{t}$ & & 0.021 & 0.026 & 0.030 \\
\hline & $\min$ & $\rho_{t}$ & & -0.048 & -0.081 & -0.112 \\
\hline & $\max$ & $\rho_{t}$ & & 0.046 & 0.076 & 0.116 \\
\hline & std dev & $C_{t}^{1}$ & $D_{t}=D^{H}$ & 0.426 & 0.723 & 0.482 \\
\hline & $\min$ & $C_{t}^{1}$ & $D_{t}=D^{H}$ & 3.605 & 3.827 & 4.235 \\
\hline & $\max$ & $C_{t}^{1}$ & $D_{t}=D^{H}$ & 6.761 & 6.665 & 6.254 \\
\hline & std dev & $C_{t}^{1}$ & $D_{t}=D^{L}$ & 0.369 & 0.630 & 0.424 \\
\hline & $\min$ & $C_{t}^{1}$ & $D_{t}=D^{L}$ & 3.317 & 3.497 & 3.859 \\
\hline & $\max$ & $C_{t}^{1}$ & $D_{t}=D^{L}$ & 6.089 & 5.973 & 5.619 \\
\hline \multirow{9}{*}{$\nu=5.00$} & std dev & $\rho_{t}$ & & 0.022 & 0.028 & 0.039 \\
\hline & $\min$ & $\rho_{t}$ & & -0.053 & -0.100 & -0.152 \\
\hline & $\max$ & $\rho_{t}$ & & 0.052 & 0.095 & 0.124 \\
\hline & std dev & $C_{t}^{1}$ & $D_{t}=D^{H}$ & 0.333 & 0.565 & 0.731 \\
\hline & $\min$ & $C_{t}^{1}$ & $D_{t}=D^{H}$ & 3.607 & 3.753 & 3.959 \\
\hline & $\max$ & $C_{t}^{1}$ & $D_{t}=D^{H}$ & 6.753 & 6.718 & 6.568 \\
\hline & std dev & $C_{t}^{1}$ & $D_{t}=D^{L}$ & 0.287 & 0.491 & 0.634 \\
\hline & $\min$ & $C_{t}^{1}$ & $D_{t}=D^{L}$ & 3.320 & 3.450 & 3.643 \\
\hline & $\max$ & $C_{t}^{1}$ & $D_{t}=D^{L}$ & 6.083 & 6.024 & 5.873 \\
\hline
\end{tabular}

Table 3 Characteristics of money non-neutrality.

\begin{tabular}{llccc}
\hline$\gamma=\nu=3.25$ & $\beta=0.92$ & $\beta=0.94$ & $\beta=0.96$ \\
\hline std dev & $\rho_{t}$ & 0.026 & 0.025 & 0.024 \\
$\min$ & $\rho_{t}$ & -0.081 & -0.075 & -0.063 \\
$\max$ & $\rho_{t}$ & 0.076 & 0.070 & 0.063 \\
std dev & $C_{t}^{1} \mid D_{t}=D^{H}$ & 0.723 & 0.782 & 0.965 \\
$\min$ & $C_{t}^{1} \mid D_{t}=D^{H}$ & 3.827 & 3.840 & 3.841 \\
$\max$ & $C_{t}^{1} \mid D_{t}=D^{H}$ & 6.665 & 6.667 & 6.665 \\
$\operatorname{std} d e v$ & $C_{t}^{1} \mid D_{t}=D^{L}$ & 0.630 & 0.688 & 0.855 \\
$\min$ & $C_{t}^{1} \mid D_{t}=D^{L}$ & 3.497 & 3.501 & 3.503 \\
$\max$ & $C_{t}^{1} \mid D_{t}=D^{L}$ & 5.973 & 5.987 & 6.010 \\
\hline
\end{tabular}

initial selection of 225 portfolios from the uniform grid $\mathcal{G}^{\prime} \subset \Xi$. For each initial point, we generated 2,000 iterations and recorded all of them.

We observe that this economy is indeed ergodic. In fact, by generating 5 million iterations starting from the initial portfolio ditribution $\pi_{0}^{1}=$ 


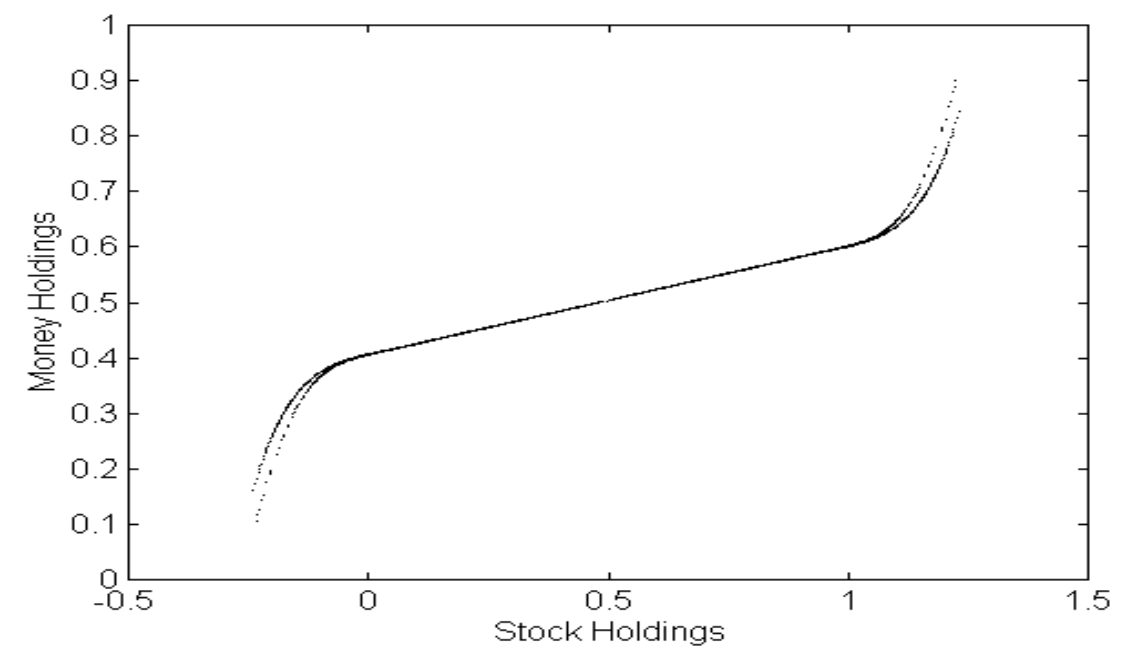

Fig. 3 REE: 450,000 observations on a grid of 225 initial portfolios.

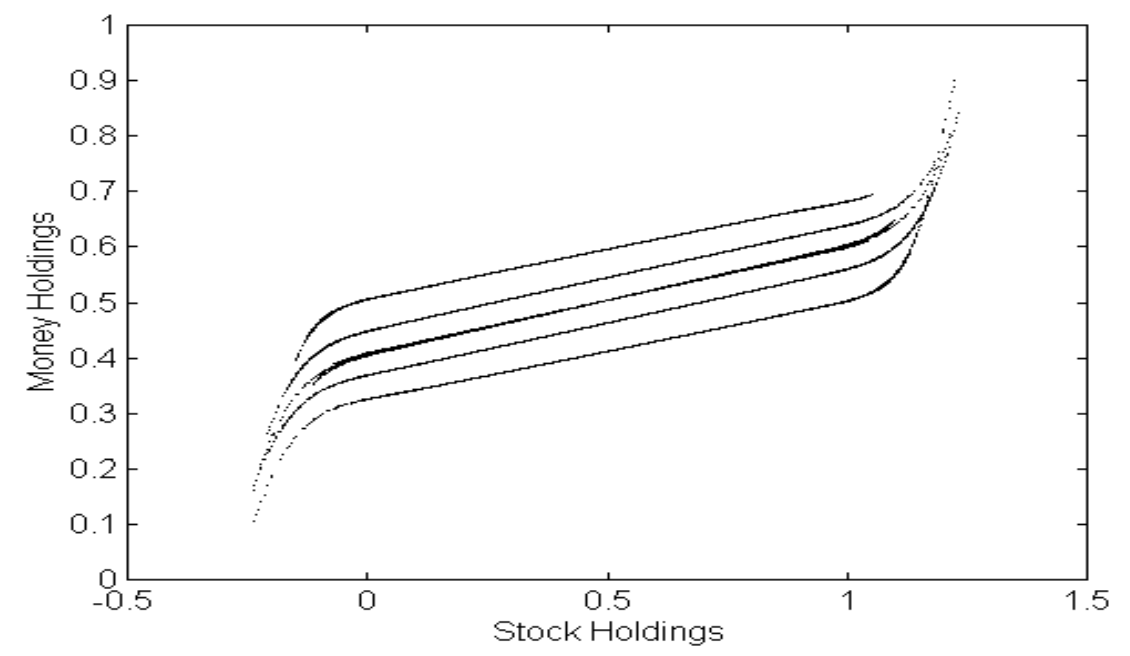

Fig. 4 RBE: 450,000 observations on a grid of 225 initial portfolios.

$(0.5,0.5)$ and then recording the last 300,000 observations we recover the ergodic set illustrated in Figure 5.

\section{Conclusions.}

In this work we have shown that dynamic non-neutrality of money is generically present in an infinite horizon RBE economy with financial assets. 


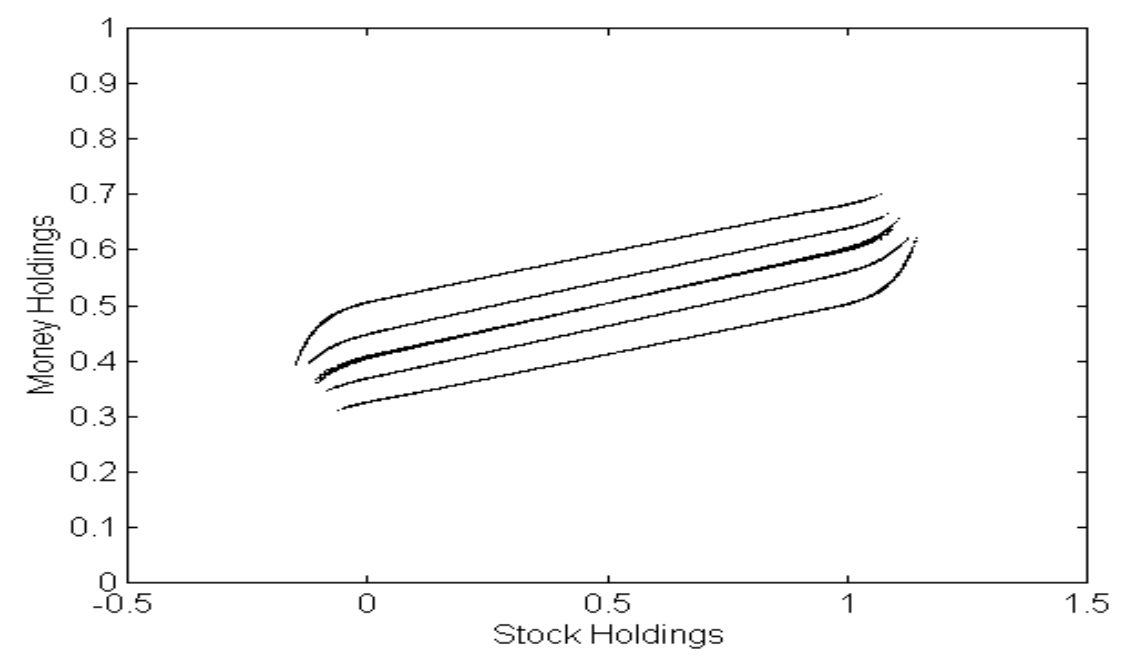

Fig. 5 The ergodic set of a RBE.

Given the expectational perspective proposed by the Theory of RBE, we have demonstrated that one of the most important factors in the emergence of money non-neutrality is played by endogenous uncertainty. This, in contrast with the Rational Expectations results of money neutrality and policy ineffectiveness, has led to a scenario in which monetary policy has an impact on the real economy and price volatility. We also showed that the heterogeneity of beliefs together with the distribution and intensity of agents' states of optimism/pessimism can amplify or reduce the real effect of monetary policy, generate Endogenous Fluctuations and give rise to a persistent effect on the long term price level volatility. Furthermore, under Rational Beliefs, states of beliefs and monetary policy have an effect on agents' portfolio allocations, hence on wealth distribution, resulting in a broader equilibrium set of trading opportunities. Under Rational Beliefs agents have different beliefs and different predictions about the effect of any particular monetary policy. And, due to the endogenously propagated mistakes of agents, monetary forces do have an impact on the real variables of the economy. We then conclude that money non-neutrality is mostly an expectations driven phenomenon. Throughout the paper we have assumed that money growth rates $x_{t}$ are observable at date $t$. Additional assumptions of asymmetry of information and/or unanticipated monetary policy have not been needed to explain the real effect of monetary policy as it is customary in the New Classical Theory. The impact of agents' state of beliefs on equilibrium dynamics has been sufficient to generate the empirically observed phenomenon of money non-neutrality. It is clear that the proposed paradigm of the Theory of Rational Beliefs has very important implications to the study of economic fluctuations and to the conduct of monetary pol- 
icy. The results obtained in the present study, Motolese [28] and Nielsen [29] represent a first step in addressing the implications of the theory proposed by Kurz [18], [19] to monetary policy. However, the issues involved are so complex that they cannot be completely and exhaustively captured by the simple model reported in this study. Further research is needed in order to obtain a satisfactory and consistent corpus of implications to monetary policy from the perspective of the Theory of Rational Beliefs.

We have also addressed the issue of how to compute numerical solutions to Rational Beliefs infinite horizon economies. In computing equilibria we have followed the work of Judd, Kubler and Schmedders [15] who developed a computational procedure based on the B-spline collocation methods of approximating the equilibrium price and portfolio policy functions. The algorithm we have used has been coded and implemented in a sequential fashion. Parallel computing techniques have been later used to solve similar models and that has drastically reduced computational time.

\section{Appendix.}

\section{A.1: Solving the system of nonlinear equations.}

The system of nonlinear equations (53)-(54) has been solved using the software package TENSOLVE, a suite of FORTRAN 77 subroutines for solving large systems of nonlinear equations using either Newton/Gauss-Newton method or a new class of methods called tensor methods. For a complete overview of the software package TENSOLVE see Bouaricha A. and Schnabel R. B. [3]. All computations have been implemented in FORTRAN on DECStations Sun Ultra Enterprise 5000/200.

\section{A.2: Approximating the equilibrium functions.}

The equilibrium functions have been approximated by a two-dimensional tensor product of B-splines of order 4 . Given a grid of knots $\left(x_{i}, y_{j}\right)$, for each sequence of knots, order $k$ B-splines are recursively defined by:

$$
B_{i}^{k}(z)=\frac{z-z_{i}}{z_{i+k}-z_{i}} B_{i}^{k-1}(z)+\frac{z_{i+k+1}-z}{z_{i+k}+1-z_{i+1}} B_{i+1}^{k-1}(z)
$$

with

$$
B_{i}^{0}(z)=\left\{\begin{array}{l}
0, z<z_{i} \\
1, z_{i} \leq z \leq z_{i-1} \\
0, z \geq z_{i}
\end{array}\right.
$$

Given a function $f(x, y)$ and points

$$
\left\{\left(\left(x_{1}, y_{1}\right), f\left(x_{1}, y_{1}\right)\right), \ldots,\left(\left(x_{n}, y_{n}\right), f\left(x_{n}, y_{n}\right)\right)\right\}
$$


the knot sequences can be chosen such that there is a unique interpolating cubic spline (i.e. a piecewise polynomial function)

$$
\hat{f}(x, y)=\sum_{i=1}^{n} \sum_{j=1}^{n} \alpha_{i j} B_{i}(x) B_{j}(y),
$$

which is a two-dimensional tensor product of B-splines. For an overview about B-splines see Judd [13].

Also De Boor [6] gives a detailed introduction into the representation of B-splines and how they can be applied to the approximation of functions. Moreover, he provides all the FORTRAN subroutines that we used for our computations.

\section{A.3: MonteCarlo simulations.}

The MonteCarlo simulations have been implemented by using the FORTRAN routine UNI to generate quasi uniform random numbers on $[0,1)$. The routine, written by Blue, Kahaner and Marsaglia, is based on a Fibonacci generator. Extensive references about the routine UNI can be found in Kahaner D., Moler C. and Nash S. [16]

\section{References}

1. Azariadis, C., Self-fulfilling Prophecies. Journal of Economic Theory 25, pp. 380-396, (1981).

2. Azariadis, C., Guesnerie, R., Sunspots and Cycles. Review of Economic Studies 53, pp. 725-737, (1986).

3. Bouaricha, A., Schnabel, R. B., TENSOLVE: A software Package for Solving Systems of Nonlinear Equations and Nonlinear Least Squares Problems Using Tensor Methods. Argonne National Laboratories, Illinois, (1994).

4. Cass, D., Shell, K., Do Sunspots Matter? Journal of Political Economy 91, pp. 193-227, (1983).

5. Chiappori, P. A., Guesnerie, R., Anticipations, Indétermination et Nonneutralité de la monnaie. Annales d'Economie et de Statistique 19, pp. 1-25, (1990).

6. De Boor, C., A Practical Guide to Splines. Springer Verlag, Berlin and New York, (1978).

7. Fisher, S., Long-Term Contracts, Rational Expectations, and the Optimal Supply Rule. Journal of Political Economy 85, pp. 191-205, (1977).

8. Fisher, S., Anticipations and the Nonneutrality of Money. Journal of Political Economy 87, pp. 225-252, (1979).

9. Harris, M., Raviv, A., Differences of Opinion Make a Horse Race. Review of Financial Studies 6, pp. 473-506, (1993).

10. Harrison, M., Kreps, D., Speculative Investor Behavior in a Stock Market with Heterogeneous Expectations. Quarterly Journal of Economics 92, pp. 323-336, (1978). 
11. Heaton, J., Lucas, D. J., Evaluating the Effects of Incomplete Markets on Risk Sharing and Asset Pricing. Journal of Political Economy 104, pp. 443487, (1996).

12. Hume, D., Of Money, (1752). Reprinted in: Rotwein, E. (ed.) Writing on Economics. University of Wisconsin Press, Madison, (1970).

13. Judd, K. J., Numerical Methods in Economics. MIT press, Cambridge, Massachussets, (1998).

14. Judd, K. J., Kubler, F., Schmedders, K., Computing Equilibria in Infinite Horizon Finance Economies: the Case of One Asset. Mimeo, Hoover Institution, Stanford University, Stanford, CA, (1997).

15. Judd, K. J., Kubler, F., Schmedders, K., Incomplete Asset Markets with Heterogeneous Tastes and Idiosyncratic Income. Mimeo, Hoover Institution, Stanford University, Stanford, CA, (1997).

16. Kahaner, D., Moler, C., Nash, S., Numerical Methods and Software. Prentice-Hall, New Jersey, (1989).

17. Kurz, M., The Kesten-Stigum Model and the Treatment of Uncertainty in Equilibrium Theory. In: Balch, M.S., McFadden, D.L., Wu, S.Y. (eds.), Essays on Economic Behavior Under Uncertainty, pp. 389-399, North Holland, Amsterdam, (1974).

18. Kurz, M., On the Structure and Diversity of Rational Beliefs. Economic Theory 4, pp. 877-900, (1994).

19. Kurz, M., On Rational Belief Equilibria. Economic Theory 4, pp. 859-876, (1994).

20. Kurz, M.(ed), Endogenous Economic Fluctuations: Studies in the Theory of Rational Belief. Studies in Economic Theory No. 6, Springer-Verlag, Berlin and New York, (1997).

21. Kurz, M., Endogenous Uncertainty: A Unified View of Market Volatility. Manuscript, Department of Economics, Stanford University, (1997).

22. Kurz, M., Social States of Belief and the Determinant of the Equity Risk Premium in a Rational Belief Equilibrium. In: Abramovich, Y. A., Avgerinos, E. and Yannelis, N. C., (eds), Functional Analysis and Economic Theory. Springer series in Applied mathematics and Operation Research, pp. 171220, Springer-Verlag, Berlin and New York, (1998).

23. Kurz, M., Motolese, M., Endogenous Uncertainty and Market Volatility. Working Paper 99-005, department of Economics, Stanford University, Stanford, CA, (2000).

24. Kurz, M., Schneider, M., Coordination and Correlation in Markov Rational Belief Equilibria. Economic Theory 8, pp. 489-520, (1996).

25. Lucas, Jr., R., Expectations and the Neutrality of Money. Journal of Economic Theory 4, pp. 103-124, (1972).

26. Lucas, Jr., R., Asset Prices in an Exchange Economy. Econometrica 46, pp. 1429-1445, (1978).

27. Lucas, Jr., R., Equilibrium in a Pure Currency Economy. Economic Inquiry XVIII, pp. 203-220, (1980).

28. Motolese, M., Dynamic Non-Neutrality of Money under Rational Beliefs: the Role of Endogenous Uncertainty. A Ph.D dissertation submitted to the University of Bologna, (November 1998).

29. Nielsen, C. K., Floating Exchange Rates versus a Monetary Union under Rational Beliefs: the Role of Endogenous Uncertainty. Mimeo, Institute of Economics, University of Copenhagen, (1997). 
30. Phelps, E., Taylor, J., Stabilizing Powers of Monetary Policy Under Rational Expectations. Journal of Political Economy 85, pp. 163-190, (1977).

31. Sargent, T. J., Wallace, N., Rational Expectations, the Optimal Monetary Instrument and the Optimal Money Supply Rule. Journal of Political Economy 83, pp. 241-254, (1975).

32. Telmer, C. I., Asset-Pricing Puzzles in Incomplete Markets. Journal of Finance 48, pp.1803-1832, (1993).

33. Varian, H. R., Differences of Opinion in Financial Markets. In: Stone, C. C., (ed.), Financial Risk: Theory, Evidence and Implications. Proceeding of the 11th Annual Economic Policy Conference of the Federal Reserve Bank of St. Louis, Kluwer Academic Publishers, Boston, 1989. 